\title{
MIXED FORMULATIONS FOR A CLASS OF VARIATIONAL INEQUALITIES*
}

\author{
Leila Slimane ${ }^{1}$, AbDerrahmane Bendali $^{2}$ and Patrick Laborde ${ }^{3}$
}

\begin{abstract}
A general setting is proposed for the mixed finite element approximations of elliptic differential problems involving a unilateral boundary condition. The treatment covers the Signorini problem as well as the unilateral contact problem with or without friction. Existence, uniqueness for both the continuous and the discrete problem as well as error estimates are established in a general framework. As an application, the approximation of the Signorini problem by the lowest order mixed finite element method of Raviart-Thomas is proved to converge with a quasi-optimal error bound.
\end{abstract}

Mathematics Subject Classification. 35J85, 76M30.

Received: January 31, 2003.

\section{INTRODUCTION}

Mixed finite element methods are generally used as conservative schemes in the approximation of elliptic boundary-value problems $[5,23]$. Their robustness is presently well-established in the treatment of numerical locking effects occurring in the approximation of stiff problems as, for instance, those including rapidly varying coefficients or those in structural mechanics involving stiffeners [7,8,22]. These methods also give a correct way to construct stable numerical schemes for flow and nearly incompressible material problems [5]. Therefore, it is desirable to extend this kind of numerical techniques to variational inequalities, in particular to those involved in stiff transmission problems with Signorini boundary conditions and in unilateral contact problems in nearly incompressible elasticity. Some previous works $(c f .[6,14,15,26])$ have already been devoted to such an extension but in a restrictive way only. Other saddle point-like formulations for inequalities have also been considered $[2,10,18]$ but without dealing with the interior equilibrium conditions.

Generally, before being solved by a mixed finite element method, a boundary-value problem must be first set in a suitable variational form: the dual mixed formulation (e.g., [5, 23]). For the Laplace equation with a Signorini boundary condition and the above mentioned unilateral contact problems, we will see below that this

\footnotetext{
Keywords and phrases. Variational inequalities, unilateral problems, Signorini problem, contact problems, mixed finite element methods, elliptic PDE.

* This work is a part of L. Slimane's Ph.D. thesis which has been jointly supported by the French Ministry of Foreign Affairs and the Algerian Government and has been performed at INSA of Toulouse within MIP laboratory.

1 Université de Moncton, Campus de Shippagen, 218, boulevard J.-D. Gauthier, Shippagen, Nouveau Brunswick, E831P6, Canada.

2 Laboratoire MIP, UMR-CNRS 5640, INSA de Toulouse, 135 Avenue de Rangueil, 31077, Toulouse Cedex 4, France.

e-mail: bendali@gmm.insa-tlse.fr

3 Laboratoire MIP, UMR-CNRS 5640, Université Toulouse 3, 118 Route de Narbonne, 31062 Toulouse Cedex 4, France.

e-mail: laborde@mip.ups-tlse.fr
} 
formulation can be viewed as a special case of the following common framework

$$
\begin{cases}(p, u) \in K \times \Lambda, & \\ a(p, q-p)+b(q-p, u) & \geq L(q-p), \quad \forall q \in K, \\ b(p, v-u) & \leq \chi(v-u), \quad \forall v \in \Lambda .\end{cases}
$$

Here, $K$ and $\Lambda$ are two closed convex subsets of two Hilbert spaces $X$ and $M$, containing $0_{X}$ and $0_{M}$, the zeros of $X$ and $M$ respectively, while $a: X \times X \rightarrow \mathbb{R}, b: X \times M \rightarrow \mathbb{R}$, and $L: X \rightarrow \mathbb{R}, \chi: M \rightarrow \mathbb{R}$ denote given continuous bilinear and linear forms.

When the following conditions are fulfilled: $K$ is equal to $X, \Lambda$ is a cone and the bilinear form $a$ is coercive on the whole space $X$, the existence and the uniqueness of $(p, u)$ are well-known [14]. Observe that, for $K=X$, the first inequality actually reduces to an equation. When the form $a$ is symmetric, the problem can be handled by standard convex optimization techniques $[12,17]$ since then, it can be equivalently written in the form of the following saddle point problem

$$
\left\{\begin{array}{l}
(p, u) \in K \times \Lambda, \\
\mathcal{L}(p, v) \leq \mathcal{L}(p, u) \leq \mathcal{L}(q, u), \quad \forall q \in K, \forall v \in \Lambda
\end{array}\right.
$$

where the Lagrangian $\mathcal{L}(q, v)$ is defined by $\mathcal{L}(q, v):=\frac{1}{2} a(q, q)-L(q)+b(q, v)-\chi(v)$. However, none of these approaches remain valid when the form $a$ is non symmetric and is coercive on a strict subspace of $X$ only. In the linear case, that is, when each inequality of problem (1) reduces to an equation, it is well-known, that the well-posedness of the variational problem, as well as the stability and the approximation properties of the discrete problem, are ensured by Brezzi's conditions [5] which mainly consist in a Brezzi-Babuška condition on $b$ and a coerciveness property for $a$, generally on a strict subspace $V$ of $X$ only, directly related to the second (in)equality:

$$
V:=\{q \in X ; b(q, v)=0, \forall v \in M\} .
$$

We shall show below how these conditions can be adapted to extend the above conclusions to problem (1). Parts of the results presented in this paper have been announced in [25].

The outline of the paper is as follows. In Section 1, we consider some examples of problems involving a unilateral boundary condition and work out various variational formulations for them, well suited for their approximation by a mixed or a mixed-hybrid finite element method. In Section 2, we will see that all these formulations can be seen as special instances of problem (1). In particular, we shall bring out some properties, they share, to set conditions yielding the existence of a solution to problem (1) and its uniqueness. Furthermore, in this general setting, we devise sufficient conditions implying that the discrete approximation of the mixed variational formulation is well-posed and derive a bound for the related error. Section 3 is devoted to a partial extension of the previous results to the case where the second inequality in system (1) involves a third bilinear form as this can be encountered, for instance, in robust approximations of problems in elasticity involving nearly incompressible materials [5]. Finally, in Section 4, the general study of Section 2 is applied to prove that the mixed approximation of the Signorini problem, by the Raviart-Thomas finite element method of the lowest order, converges with a quasi-optimal error.

\section{Mixed FORMUlations of SOME Unilateral BOUNDARY-VALUE PROBLEMS}

In this section, we give some examples of unilateral boundary-value problems which have not apparently been dealt with using mixed finite-element methods yet. These examples will mainly permit us to introduce the adaptations of the usual Brezzi's conditions made necessary by the present context.

In all the sequel, for the sake of simplicity, we limit the exposure to the case where $\Omega$ is a bounded domain of the plane. Its boundary $\partial \Omega$ is assumed to be at least Lipschitz and is decomposed as a non-overlapping union of three subsets $\Gamma_{D}, \Gamma_{N}$ and $\Gamma_{C} ;$ parts $\Gamma_{D}$ and $\Gamma_{C}$ of the boundary, respectively endowed with a Dirichlet and a 
unilateral boundary condition, are assumed to be non empty. The unit normal to $\partial \Omega$, outwardly directed to $\Omega$, is denoted by $\boldsymbol{n}$.

\subsection{Signorini problem}

Let $\boldsymbol{A}=\left(A_{i j}\right)_{1 \leq i, j \leq 2}$ be a matrix-valued function with $A_{i j} \in L^{\infty}(\Omega)$, satisfying the usual uniform ellipticity condition in $\Omega$, that is,

$$
\exists \gamma>0: \boldsymbol{\xi}^{\top} \boldsymbol{A} \boldsymbol{\xi} \geq \gamma|\boldsymbol{\xi}|^{2}, \quad \forall \boldsymbol{\xi} \in \mathbb{R}^{2}, \quad \text { a.e. in } \Omega .
$$

Given $f$ in $L^{2}(\Omega)$, the Signorini problem, related to a Laplace-type equation in $\Omega$, has the following statement: find $u \in H^{1}(\Omega)$ such that

$$
\left\{\begin{array}{l}
-\boldsymbol{\nabla} \cdot \boldsymbol{A} \boldsymbol{\nabla} u=f \text { in } \Omega, \quad u=0 \text { on } \Gamma_{D}, \boldsymbol{A} \boldsymbol{\nabla} u \cdot \boldsymbol{n}=0 \text { on } \Gamma_{N} \\
u \geq 0, \quad \boldsymbol{A} \boldsymbol{\nabla} u \cdot \boldsymbol{n} \geq 0, \quad u \boldsymbol{A} \boldsymbol{\nabla} u \cdot \boldsymbol{n}=0 \text { on } \Gamma_{C} .
\end{array}\right.
$$

Neumann and unilateral boundary conditions have a meaning in the sense of distributions on $\partial \Omega$ as this is recalled below for similar instances. Symbol $\boldsymbol{\nabla}$ is used as usual to denote the gradient $\boldsymbol{\nabla} u$ of function $u$ as well as the divergence $\boldsymbol{\nabla} \cdot \boldsymbol{q}=\partial_{1} q_{1}+\partial_{2} q_{2}$ of a vector field $\boldsymbol{q}, q_{1}$ and $q_{2}$ being its components. The notation and the functional spaces that are standard in the partial differential equation theory are used without further comment (see for instance $[5,9]$ ).

In the mixed formulations of second order scalar elliptic problems (e.g., $[5,23])$, the flux density $\boldsymbol{p}=\boldsymbol{A} \boldsymbol{\nabla} u$ is taken as the main unknown of the problem. It belongs, in a natural way, to the following Hilbert space

$$
H(\operatorname{div} ; \Omega):=\left\{\boldsymbol{q} \in L^{2}\left(\Omega ; \mathbb{R}^{2}\right) ; \nabla \cdot \boldsymbol{q} \in L^{2}(\Omega)\right\}
$$

where $L^{2}\left(\Omega ; \mathbb{R}^{2}\right)$ is defined as the space of vector fields $\boldsymbol{q}$ whose components $q_{j}$ are in $L^{2}(\Omega)$ for $j=1,2$. The unilateral boundary condition is expressed by seeking $\boldsymbol{p}$ in the following convex cone $\boldsymbol{K}$ of $H($ div; $\Omega$ ) whose elements $\boldsymbol{q}$ verify the following variational inequality

$$
\int_{\Omega} \boldsymbol{q} \cdot \boldsymbol{\nabla} v \mathrm{~d} \Omega+\int_{\Omega} v \boldsymbol{\nabla} \cdot \boldsymbol{q} \mathrm{d} \Omega \geq 0, \forall v \in H^{1}(\Omega), v=0 \text { on } \Gamma_{D}, v \geq 0 \text { on } \Gamma_{C} .
$$

The mixed dual formulation of (3) can then be stated as follows

$$
\left\{\begin{array}{lll}
(\boldsymbol{p}, u) \in \boldsymbol{K} \times L^{2}(\Omega), & & \\
a(\boldsymbol{p}, \boldsymbol{q}-\boldsymbol{p})+b(\boldsymbol{q}-\boldsymbol{p}, u) & \geq 0, & \forall \boldsymbol{q} \in \boldsymbol{K}, \\
b(\boldsymbol{p}, v) & =-\int_{\Omega} f v \mathrm{~d} \Omega, & \forall v \in L^{2}(\Omega),
\end{array}\right.
$$

where

$$
\begin{aligned}
& a(\boldsymbol{p}, \boldsymbol{q}):=\int_{\Omega} \boldsymbol{A}^{-1} \boldsymbol{p} \cdot \boldsymbol{q} \mathrm{d} \Omega, \quad \boldsymbol{p}, \boldsymbol{q} \in H(\operatorname{div} ; \Omega), \\
& b(\boldsymbol{q}, v):=\int_{\Omega} v \boldsymbol{\nabla} \cdot \boldsymbol{q} \mathrm{d} \Omega, \quad v \in L^{2}(\Omega), \boldsymbol{q} \in H(\operatorname{div} ; \Omega) .
\end{aligned}
$$

Denote by $\boldsymbol{W}$ the subspace contained in $\boldsymbol{K}$ and defined by

$$
\boldsymbol{W}:=\{\boldsymbol{q} \in \boldsymbol{K} ;-\boldsymbol{q} \in \boldsymbol{K}\},
$$

and by $\boldsymbol{V}$ the "kernel" of the bilinear form $b$, that is,

$$
\boldsymbol{V}:=\left\{\boldsymbol{q} \in H(\operatorname{div} ; \Omega) ; b(\boldsymbol{q}, v)=0, \forall v \in L^{2}(\Omega)\right\}
$$


which can also be explicitly expressed as follows

$$
\boldsymbol{V}:=\{\boldsymbol{q} \in H(\operatorname{div} ; \Omega) ; \boldsymbol{\nabla} \cdot \boldsymbol{q}=0 \text { in } \Omega\}
$$

We can thus bring out the most important feature for mixed formulation (5) of problem (3). Indeed, the bilinear form $a$ is coercive on $\boldsymbol{V}$,

$$
\exists \alpha>0: a(\boldsymbol{q}, \boldsymbol{q}) \geq \alpha\|\boldsymbol{q}\|_{H(\mathrm{div} ; \Omega)}^{2}, \forall \boldsymbol{q} \in \boldsymbol{V},
$$

but not on the whole space $H(\operatorname{div} ; \Omega)$. When restricted to $\boldsymbol{W} \times L^{2}(\Omega)$, the bilinear form $b$ satisfies the following Babuška-Brezzi inf-sup condition (see [5]):

$$
\exists \beta>0: \quad \sup _{\boldsymbol{q} \in \boldsymbol{W}} \frac{b(\boldsymbol{q}, v)}{\|\boldsymbol{q}\|_{H(\operatorname{div} ; \Omega)}} \geq \beta\|v\|_{0, \Omega}, \quad \forall v \in L^{2}(\Omega) .
$$

Note that, unless $\boldsymbol{A}(x)$ is equal to its transpose $\boldsymbol{A}^{\top}(x)$ a.e. in $\Omega$, the bilinear form $a$ is not symmetric.

\subsection{Dualization of the unilateral boundary conditions}

Let $\Gamma_{C N}$ be such it constitutes with $\Gamma_{D}$ a non-overlapping decomposition of $\partial \Omega$. The following Sobolev space

$$
H_{00}^{1 / 2}\left(\Gamma_{C N}\right):=\left\{\mu \in H^{1 / 2}(\partial \Omega) ; \mu=0 \text { on } \Gamma_{D}\right\}
$$

is generally considered to characterize traces on $\Gamma_{C N}$ of functions in $H^{1}(\Omega)$ which furthermore vanish in a stable way at the end-points of $\Gamma_{C N}$.

Weakening the unilateral condition on $\Gamma_{C}$, we get another equivalent mixed variational formulation to problem (3)

$$
\begin{cases}(\boldsymbol{p},(u, \lambda)) \in H(\operatorname{div} ; \Omega) \times \boldsymbol{\Lambda}, & \\ a(\boldsymbol{p}, \boldsymbol{q})+b(\boldsymbol{q},(u, \lambda))=0, & \forall \boldsymbol{q} \in H(\operatorname{div} ; \Omega), \\ b(\boldsymbol{p},(v, \mu)-(u, \lambda)) \leq \chi((v, \mu)-(u, \lambda)), & \forall(v, \mu) \in \boldsymbol{\Lambda} .\end{cases}
$$

The convex set $\boldsymbol{\Lambda}$ consists of all $(v, \mu)$ in $L^{2}(\Omega) \times H_{00}^{1 / 2}\left(\Gamma_{C N}\right)$ such that $\mu \leq 0$ on $\Gamma_{C}$. The bilinear form $b$ and linear form $\chi$ are now respectively defined by

$$
b(\boldsymbol{q},(v, \mu)):=\int_{\Omega} v \boldsymbol{\nabla} \cdot \boldsymbol{q} \mathrm{d} \Omega+\langle\mu, \boldsymbol{q} \cdot \boldsymbol{n}\rangle_{1 / 2, \Gamma_{C N}}, \quad \chi(v, \mu)=-\int_{\Omega} f v \mathrm{~d} \Omega .
$$

Brackets $\langle\cdot, \cdot\rangle_{1 / 2, \Gamma_{C N}}$ stand for the duality pairing between $H_{00}^{1 / 2}\left(\Gamma_{C N}\right)$ and its dual $\left(H_{00}^{1 / 2}\left(\Gamma_{C N}\right)\right)^{\prime}$.

For some instances of problem (1), the subspace $V$, on which $a$ becomes a coercive bilinear form, is defined through the largest subspace $Z$ contained in the convex $\Lambda$. In this way, for the present example, we take for $\boldsymbol{Z}$ the subspace of $L^{2}(\Omega) \times H_{00}^{1 / 2}\left(\Gamma_{C N}\right)$ defined by

$$
\boldsymbol{Z}:=\{(v, \mu) \in \boldsymbol{\Lambda} ;-(v, \mu) \in \boldsymbol{\Lambda}\} .
$$

The subspace of $H(\operatorname{div} ; \Omega)$ associated to the bilinear form $b$ is hence defined by

$$
\boldsymbol{V}:=\{\boldsymbol{q} \in H(\operatorname{div} ; \Omega) ; \quad b(\boldsymbol{q},(v, \mu))=0, \quad \forall(v, \mu) \in \boldsymbol{Z}\} .
$$

This is nothing else but the following subspace of $H(\operatorname{div} ; \Omega)$

$$
\boldsymbol{V}:=\left\{\boldsymbol{q} \in H(\operatorname{div} ; \Omega) ; \quad \boldsymbol{\nabla} \cdot \boldsymbol{q}=0 \text { in } \Omega, \boldsymbol{q} \cdot \boldsymbol{n}=0 \text { on } \Gamma_{N}\right\}
$$


The bilinear form $a$ is obviously coercive on $\boldsymbol{V}$. The other Brezzi's condition is established in the following lemma.

Lemma 1.1. There exists a positive constant $\beta$, independent of $(v, \mu)$ in $\boldsymbol{\Lambda}$, such that

$$
\sup _{\boldsymbol{q} \in H(\operatorname{div} ; \Omega)} \frac{b(\boldsymbol{q},(v, \mu))}{\|\boldsymbol{q}\|_{H(\operatorname{div} ; \Omega)}} \geq \beta\left(\|v\|_{0, \Omega}^{2}+\|\mu\|_{H_{00}^{1 / 2}\left(\Gamma_{C N}\right)}^{2}\right)^{1 / 2}, \quad \forall(v, \mu) \in \mathbf{\Lambda} .
$$

Proof. See [24] for a proof.

\subsection{Contact problem}

Consider the following unilateral contact problem in plane elasticity $[11,16,17]$. An elastic body, represented by the domain $\Omega$ in its initial configuration, has a displacement field $\boldsymbol{u}$ under the action of a density $\boldsymbol{f}$ of external volume forces. The stress and the linearized strain tensor fields are denoted in a usual way by $\boldsymbol{\sigma}:=\left\{\sigma_{i j}\right\}_{i, j=1,2}$ and $\boldsymbol{\epsilon}(\boldsymbol{u}):=\left\{\varepsilon_{i j}(\boldsymbol{u})\right\}_{i, j=1,2}$ respectively. Finally, the constitutive relations are given through a 4th-order symmetric tensor $\boldsymbol{A}$, assumed to satisfy the standard ellipticity condition. The governing equations for the problem are then

$$
\boldsymbol{\sigma}=\boldsymbol{A} \boldsymbol{\epsilon}(\boldsymbol{u}),-\operatorname{Div} \boldsymbol{\sigma}=\boldsymbol{f} \text { in } \Omega, \boldsymbol{u}=0 \text { on } \Gamma_{D}, \boldsymbol{\sigma} \boldsymbol{n}=0 \text { on } \Gamma_{N}
$$

where $\mathbf{D i v} \boldsymbol{\sigma}$ stands for the vector field the components of which are $(\mathbf{D i v} \boldsymbol{\sigma})_{i}:=\sum_{j=1}^{2} \partial_{j} \sigma_{i j}, i=1,2$. Traction forces on $\Gamma_{N}$ can also be considered without any supplementary difficulty.

On $\Gamma_{C}$, using the standard decomposition of stress and displacement vectors on $\partial \Omega$ in a tangential and a normal component

$$
\boldsymbol{\sigma} \boldsymbol{n}=\boldsymbol{\sigma}_{T}+\sigma_{N} \boldsymbol{n}, \quad \boldsymbol{u}=\boldsymbol{u}_{T}+u_{N} \boldsymbol{n},
$$

the unilateral boundary condition can either be a friction-less condition

$$
u_{N} \leq 0, \sigma_{N} \leq 0, u_{N} \sigma_{N}=0, \text { and } \boldsymbol{\sigma}_{T}=0,
$$

or a Tresca friction condition

$$
\left\{\begin{array}{l}
u_{N} \leq 0, \sigma_{N} \leq 0, u_{N} \sigma_{N}=0,\left|\boldsymbol{\sigma}_{T}\right| \leq s \\
\text { if }\left|\boldsymbol{\sigma}_{T}\right|<s \text { then } \boldsymbol{u}_{T}=0, \text { else } \boldsymbol{u}_{T}=-\lambda \boldsymbol{\sigma}_{T} \text { with } \lambda \geq 0 .
\end{array}\right.
$$

The threshold $s$ is assumed to be known a priori and such that $s \in L^{\infty}\left(\Gamma_{C}\right)$ and $s \geq 0$.

Recall that $H_{\text {sym }}(\mathbf{D i v} ; \Omega)$ is the subspace of symmetric tensors $\boldsymbol{\tau}$, i.e., such that $\tau_{12}=\tau_{21}$, that belong to

$$
H(\operatorname{Div} ; \Omega):=\left\{\boldsymbol{\tau} \in L^{2}\left(\Omega ; \mathbb{R}^{2,2}\right) ; \operatorname{Div} \boldsymbol{\tau} \in L^{2}\left(\Omega ; \mathbb{R}^{2}\right)\right\} .
$$

Let $\boldsymbol{K}$ be the convex set of all $\boldsymbol{\tau}$ in $H_{\text {sym }}($ Div; $\Omega)$ satisfying

$$
\langle\boldsymbol{\tau} \boldsymbol{n}, \boldsymbol{\varphi}\rangle_{1 / 2, \Gamma_{C N}} \leq 0, \forall \boldsymbol{\varphi} \in H_{00}^{1 / 2}\left(\Gamma_{C N} ; \mathbb{R}^{2}\right), \varphi_{N} \geq 0 \text { on } \Gamma_{C},
$$

in the friction-less case (7), or

$$
\langle\boldsymbol{\tau} \boldsymbol{n}, \boldsymbol{\varphi}\rangle_{1 / 2, \Gamma_{C N}} \leq \int_{\Gamma_{C}} s\left|\boldsymbol{\varphi}_{T}\right| \mathrm{d} \Gamma_{C}, \forall \boldsymbol{\varphi} \in H_{00}^{1 / 2}\left(\Gamma_{C N} ; \mathbb{R}^{2}\right), \varphi_{N} \geq 0 \text { on } \Gamma_{C},
$$


when dealing with Tresca condition (8). The duality pairing is defined accordingly to the scalar case. It can easily be seen that the two instances of the above elasticity problem have the following dual mixed formulation:

$$
\begin{cases}(\boldsymbol{\sigma}, \boldsymbol{u}) \in \boldsymbol{K} \times L^{2}\left(\Omega ; \mathbb{R}^{2}\right), & \\ a(\boldsymbol{\sigma}, \boldsymbol{\tau}-\boldsymbol{\sigma})+b(\boldsymbol{\tau}-\boldsymbol{\sigma}, \boldsymbol{u}) \geq 0, & \forall \boldsymbol{\tau} \in \boldsymbol{K}, \\ b(\boldsymbol{\sigma}, \boldsymbol{v})=\chi(\boldsymbol{v}), & \forall \boldsymbol{v} \in L^{2}\left(\Omega ; \mathbb{R}^{2}\right),\end{cases}
$$

where

$$
a(\boldsymbol{\sigma}, \boldsymbol{\tau})=\int_{\Omega} \boldsymbol{A}^{-1} \boldsymbol{\sigma}: \boldsymbol{\tau} \mathrm{d} \Omega, \quad b(\boldsymbol{\sigma}, \boldsymbol{v})=\int_{\Omega} \boldsymbol{v} \cdot \operatorname{Div} \boldsymbol{\sigma} \mathrm{d} \Omega, \quad \chi(\boldsymbol{v})=-\int_{\Omega} \boldsymbol{f} \cdot \boldsymbol{v} \mathrm{d} \Omega,
$$

and $\boldsymbol{\sigma}: \boldsymbol{\tau}$ stands for the usual scalar product of $\boldsymbol{\sigma}$ and $\boldsymbol{\tau}$.

One can immediately verify that the bilinear form $a$ is coercive on the subspace

$$
\boldsymbol{V}:=\left\{\boldsymbol{\tau} \in H_{\mathrm{sym}}(\mathbf{D i v} ; \Omega) ; b(\boldsymbol{\tau}, \boldsymbol{v})=0, \forall \boldsymbol{v} \in L^{2}\left(\Omega ; \mathbb{R}^{2}\right)\right\}
$$

which here can also be defined by $\boldsymbol{V}:=\left\{\boldsymbol{\tau} \in H_{\text {sym }}(\operatorname{Div} ; \Omega) ; \operatorname{Div} \boldsymbol{\tau}=0\right.$ in $\left.\Omega\right\}$.

Furthermore, denoting by

$$
\boldsymbol{W}:=\left\{\boldsymbol{\tau} \in H_{\mathrm{sym}}(\operatorname{Div} ; \Omega) ;\langle\boldsymbol{\tau} \boldsymbol{n}, \boldsymbol{\varphi}\rangle_{1 / 2, \partial \Omega}=0, \forall \boldsymbol{\varphi} \in H_{00}^{1 / 2}\left(\Gamma_{C N} ; \mathbb{R}^{2}\right)\right\}
$$

we readily obtain the following inf-sup condition (see [5])

$$
\exists \beta>0: \sup _{\boldsymbol{\tau} \in \boldsymbol{W}} \frac{b(\boldsymbol{\tau}, \boldsymbol{v})}{\|\boldsymbol{\tau}\|_{H(\operatorname{Div} ; \Omega)}} \geq \beta\|\boldsymbol{v}\|_{0, \Omega}, \quad \forall \boldsymbol{v} \in L^{2}\left(\Omega ; \mathbb{R}^{2}\right) .
$$

A procedure, similar to that presented above for the Signorini problem, is used to dualize the Tresca condition in [2]. A similar problem related to a frictionless unilateral contact problem of two elastic bodies has been considered in $[10,18]$. In all these works, the stresses are removed from the formulation. The mixed character of the problem remains in the treatment of the boundary condition only. This avoids the main difficulty of the equilibrium formulations where the coercivity of the bilinear form $a$ is satisfied on the subspace $\boldsymbol{V}$ only.

The first step in the construction of a stable mixed finite element scheme for the above problems is to check that each of problems $(5,6)$ and $(9)$ is well-posed. All the above formulations can be studied within the general setting (1).

\section{THE ABSTRACT FRAMEWORK}

In the light of the above examples, we are led to study the existence and the uniqueness of a solution to problem (1). Recall that, in the linear case, it is Brezzi's conditions which guarantee that extension (1) of saddle point problem (2) admits one and only one solution [5]. We must hence determine conditions that will play the same role for the system of variational inequalities. They will be derived from the above observations on the Signorini and the contact problems.

\subsection{The continuous problem}

Before beginning the study of problem (1), we first prove the following simple but crucial properties which will be used several times below.

Lemma 2.1. Let $E$ be a normed space and $F \subset K$ respectively a subspace and a closed convex set of $E$. Then, the following property holds

$$
u+v \in K \text { whenever } u \in K \text { and } v \in F \text {. }
$$


Furthermore, if $\ell$ is a linear form on $E$ and $u \in K$ are such that $\ell(v-u) \geq 0, \forall v \in K$, then $\ell$ vanishes on $F$, that is,

$$
\ell(w)=0, \quad \forall w \in F
$$

Proof. To establish (10), it is sufficient to remark that for $0<t<1,(1-t) u+t(v / t) \in K$ and to let $t \rightarrow 0$. Property (11) is obtained by taking $v=u \pm w$.

The bilinear form $a$ is not necessarily coercive on the whole space $X$ but it is always assumed to be at least non negative, that is,

$$
a(q, q) \geq 0, \quad \forall q \in X
$$

This assumption is satisfied in almost all mixed formulations of unilateral boundary-value problems.

We first define an extension of the first Brezzi's condition, that is, the inf-sup condition. This is done by assuming that it exists a closed subspace $W \subset K$ and a constant $\beta>0$ such that

$$
\exists \beta>0: \quad \sup _{q \in W} \frac{b(q, v)}{|q|_{X}} \geq \beta|v|_{M}, \quad \forall v \in M
$$

where we have denoted by $|\cdot|_{X}$ and by $|\cdot|_{M}$ the norms in $X$ and $M$ respectively.

Next, the second Brezzi's condition is extended as follows. It is assumed that there exists a subspace $Z$ contained in the convex set $\Lambda$ such that, for

$$
V:=\{q \in X ; b(q, v)=0, \quad \forall v \in Z\}
$$

the following coercivity estimate holds

$$
\exists \alpha>0: a(q, q) \geq \alpha|q|_{X}^{2}, \quad \forall q \in V .
$$

The existence of a solution to problem (1) is based on an a priori estimate which, surprisingly enough, is exactly that well-known in the linear case [5]. Indeed, this estimate gives a uniform bound which can be also useful for other purposes. The notation $|L|_{X^{\prime}}$ and $|\chi|_{M^{\prime}}$ is respectively used to denote the dual norm of $L$ in $X^{\prime}$ relatively to the norm in $X$ and similarly for $\chi$.

Finally, defining $M_{a}$ and $M_{b}$ as the continuity constants of $a$ and $b$ respectively, i.e., the smallest constants such that

$$
|a(p, q)| \leq M_{a}|p|_{X}|q|_{X}, \text { for all } p \text { and } q \text { in } X, \quad|b(q, v)| \leq M_{b}|q|_{X}|v|_{M} \text {, for all } q \text { in } X \text { and } v \text { in } M
$$

in all the rest of this subsection, we denote by $C$ various constants, whose values may change from place to place, depending only on $M_{a}, M_{b}, 1 / \alpha$ and $1 / \beta$, and remaining bounded when these quantities are varying in a bounded set.

Lemma 2.2. Assume that conditions (13) and (14) hold. Then, any solution ( $p, u)$ to problem (1) satisfies the following bound

$$
|p|_{X}+|u|_{M} \leq C\left(|L|_{X^{\prime}}+|\chi|_{M^{\prime}}\right)
$$

Proof. We make use again of the inf-sup condition to establish that there exists $r \in W$ such that

$$
b(r, v)=\chi(v), \quad \forall v \in M,
$$

which furthermore can be controlled by $|\chi|_{M^{\prime}}:|r|_{X} \leq C|\chi|_{M^{\prime}}$. In view of the first inequality in (1), we get $a(p, r-p)+b(r-p, u) \geq L(r-p)$, which, since the second one can be written as $b(r-p, u) \leq 0$, yields

$$
a(p-r, p-r) \leq L(p-r)-a(r, p-r) .
$$


As $p-r \in V$ and $a$ is coercive on $V$, it follows that

$$
|p|_{X} \leq C\left(|L|_{X^{\prime}}+|\chi|_{M^{\prime}}\right) .
$$

Once more, it is the inf-sup condition which gives a similar estimate on $u$

$$
|u|_{M} \leq C\left(|L|_{X^{\prime}}+|p|_{X}\right)
$$

and completes the proof of the lemma.

In this context, the following theorem holds.

Theorem 2.3. Under the general conditions of continuity above assumed on bilinear forms $a$ and $b$ and on linear forms $L$ and $\chi$, condition (12), Brezzi's inf-sup condition (13) and coercivity condition (14) (in particular, by no mean a has to be symmetric), problem (1) admits one and only one solution.

Proof. It is done in four steps.

First step. The first ingredient is to put problem (1) in another equivalent form as that used in [14]. Toward this end, we consider the product space $\mathcal{H}=X \times M$, equipped with its Hilbertian norm

$$
|Q|_{\mathcal{H}}=\left(|q|_{X}^{2}+|v|_{M}^{2}\right)^{1 / 2}, \quad Q=(q, v) \in \mathcal{H},
$$

along with the continuous bilinear form $\mathcal{A}: \mathcal{H} \times \mathcal{H} \longrightarrow \mathbb{R}$ defined by

$$
\mathcal{A}(P, Q)=a(p, q)+b(q, u)-b(p, v), \quad P=(p, u) \in \mathcal{H}, Q=(q, v) \in \mathcal{H},
$$

and the continuous linear form $\mathcal{F}: \mathcal{H} \longrightarrow \mathbb{R}$ given by

$$
\mathcal{F}(Q)=L(q)-\chi(v), \quad Q=(q, v) \in \mathcal{H} .
$$

Note that $\mathcal{A}$ is not symmetric even if it is the case for the bilinear form $a$. It can then be readily seen that problem (1) is equivalent to the following single variational inequality

$$
\left\{\begin{array}{l}
P=(p, u) \in K \times \Lambda, \\
\mathcal{A}(P, Q-P) \geq \mathcal{F}(Q-P), \quad \forall Q \in K \times \Lambda .
\end{array}\right.
$$

It is worth noting that when the bilinear form $\mathcal{A}$ is coercive on the whole space $\mathcal{H}$, Stampacchia's theorem ensures that problem (15) has a solution (e.g., $[4,19])$. Since this is not true here, we use a perturbation technique based on the consideration of a bilinear form $\mathcal{A}_{\delta}$ depending on a small parameter $\delta>0$ defined by

$$
\mathcal{A}_{\delta}(P, Q)=\mathcal{A}(P, Q)+\delta(p, q)_{X}+\delta(u, v)_{M}, \quad P=(p, u) \in \mathcal{H}, Q=(q, v) \in \mathcal{H},
$$

where $(\cdot, \cdot)_{X}$ and $(\cdot, \cdot)_{M}$ respectively denote the inner product in $X$ and in $M$. Clearly, $\mathcal{A}_{\delta}$ now has the required coercivity

$$
\mathcal{A}_{\delta}(Q, Q) \geq \delta\left(|q|_{X}^{2}+|v|_{M}^{2}\right), \quad \forall Q=(q, v) \in \mathcal{H}
$$

Therefore, the parameter dependent problem

$$
\left\{\begin{array}{l}
P_{\delta}=\left(p_{\delta}, u_{\delta}\right) \in K \times \Lambda \\
\mathcal{A}_{\delta}\left(P_{\delta}, Q-P_{\delta}\right) \geq \mathcal{F}\left(Q-P_{\delta}\right), \quad \forall Q \in K \times \Lambda,
\end{array}\right.
$$

has one and only one solution. 
Second step. In this step, we show that the sequence $\left\{\left(p_{\delta}, u_{\delta}\right)\right\}_{\delta>0}$ is uniformly bounded in $\delta$. The key step in the proof relies on coercivity (14) of $a$ on $V$ which gives that

$$
a_{\delta}(q, q):=a(q, q)+\delta(q, q)_{X} \geq \alpha|q|_{X}^{2}, \quad \forall q \in V .
$$

Moving $\delta\left(u_{\delta}, v-u_{\delta}\right)$ to the right hand side of the second inequality, Lemma (2.2) then yields

$$
\left|p_{\delta}\right|_{X}+\left|u_{\delta}\right|_{M} \leq C\left(|L|_{X^{\prime}}+|\chi|_{M^{\prime}}+\delta\left|u_{\delta}\right|_{M}\right)
$$

Since $\delta$ is destined to go to 0 , this leads to the announced bound.

Third step. Therefore, possibly passing to a sub-sequence, we can assume that $\left\{\left(p_{\delta}, p_{\delta}\right)\right\}_{\delta>0}$ converges weakly in $\mathcal{H}$ to an element $(p, u) \in K \times \Lambda$ as well as $a\left(p_{\delta}, p_{\delta}\right)$ to $a_{*}$ as $\delta \rightarrow 0$. Our aim now is to show that the limit $(p, u)$ satisfies $(1)$. Since $P_{\delta}=\left(p_{\delta}, u_{\delta}\right)$ verifies $(16)$, we have

$$
\mathcal{A}_{\delta}\left(P_{\delta}, Q\right)-a\left(p_{\delta}, p_{\delta}\right)-\delta\left(\left|p_{\delta}\right|_{X}^{2}+\left|u_{\delta}\right|_{M}^{2}\right) \geq \mathcal{F}\left(Q-P_{\delta}\right), \quad \forall Q \in K \times \Lambda
$$

Making use of the fact that the bilinear form $a$ is non negative (12), we get that $\lim _{\delta \rightarrow 0} a\left(p_{\delta}, p_{\delta}\right)=a_{*} \geq a(p, p)$. Letting $\delta \rightarrow 0$, we readily obtain

$$
\mathcal{A}(P, Q)-a(p, p) \geq \mathcal{F}(Q-P), \quad \forall Q \in K \times \Lambda
$$

As $\mathcal{A}(P, Q)-a(p, p)=\mathcal{A}(P, Q-P)$, the above inequality can be written in the following form

$$
\mathcal{A}(P, Q-P) \geq \mathcal{F}(Q-P), \quad \forall Q \in K \times \Lambda
$$

which exactly expresses that $(p, u)$ is a solution to $(1)$.

Fourth step. Assume that problem (1) admits two solutions, $\left(p_{1}, u_{1}\right)$ and $\left(p_{2}, u_{2}\right)$. Hence, by suitably choosing the test functions when successively considering each of these solutions, we get

$$
\begin{aligned}
& a\left(p_{1}, p_{2}-p_{1}\right)+b\left(p_{2}-p_{1}, u_{1}\right) \geq L\left(p_{2}-p_{1}\right) \\
& a\left(p_{2}, p_{1}-p_{2}\right)+b\left(p_{1}-p_{2}, u_{2}\right) \geq L\left(p_{1}-p_{2}\right) .
\end{aligned}
$$

Adding these two inequalities, we first obtain

$$
a\left(p_{1}-p_{2}, p_{1}-p_{2}\right)+b\left(p_{1}-p_{2}, u_{1}-u_{2}\right) \leq 0
$$

On the other hand, we have

$$
b\left(p_{1}, u_{1}-u_{2}\right) \geq \chi\left(u_{1}-u_{2}\right), \quad b\left(p_{2}, u_{2}-u_{1}\right) \geq \chi\left(u_{2}-u_{1}\right) .
$$

The two above inequalities yield $b\left(p_{1}-p_{2}, u_{1}-u_{2}\right) \geq 0$. Coming back to (17), we obtain $a\left(p_{1}-p_{2}, p_{1}-p_{2}\right) \leq 0$. Lemma 2.1 gives that $b\left(p_{j}, w\right)=\chi(w), \forall w \in Z$, for $j=1,2$, hence, yielding $p_{1}-p_{2} \in V$. Using the coercivity of $a$, we conclude that $p_{1}=p_{2}$. It remains to prove that $u_{1}=u_{2}$. To do so, it is sufficient to observe that (11) gives

which directly yields

$$
a\left(p_{1}, q\right)+b\left(q, u_{1}\right)=L(q) \text { and } a\left(p_{1}, q\right)+b\left(q, u_{2}\right)=L(q), \quad \forall q \in W,
$$

$$
b\left(q, u_{1}-u_{2}\right)=0, \quad \forall q \in W .
$$

The conclusion is a direct consequence of inf-sup condition (13). This completes the proof of the theorem. 
Remark 2.4. Observe that, when $a$ is coercive on the whole space $X$, that is,

$$
\exists \alpha>0: a(q, q) \geq \alpha|q|_{X}^{2}, \quad \forall q \in X
$$

it is automatically non negative and condition (14) becomes irrelevant. When this property holds, some points of the proofs become much more easier.

Since the above unilateral boundary-value problems can be seen as concrete particular cases of general problem (1), we have indeed proved the following result.

Corollary 2.5. Each of problems $(5,6)$ and (9) admits one and only one solution satisfying the general bound of Lemma 2.2.

\subsection{The discrete problem and error estimates}

Let $X_{h}$ and $M_{h}$ be two finite dimensional sub-spaces of $X$ and $M$, and $K_{h}, \Lambda_{h}$ two closed convex subsets of $X_{h}$ and $M_{h}$ respectively. As for the continuous case, we assume that $0_{X} \in K_{h}$ and $0_{M} \in \Lambda_{h}$. As usual, $h$ denotes the discretization parameter and is destined to tend to zero. Note that neither $K_{h}$ is assumed to be included in $K$ nor that $\Lambda_{h}$ is a subset of $\Lambda$. The discrete problem can now be stated as follows

$$
\begin{cases}\left(p_{h}, u_{h}\right) \in K_{h} \times \Lambda_{h}, & \\ a\left(p_{h}, q_{h}-p_{h}\right)+b\left(q_{h}-p_{h}, u_{h}\right) \geq L\left(q_{h}-p_{h}\right), & \forall q_{h} \in K_{h}, \\ b\left(p_{h}, v_{h}-u_{h}\right) \leq \chi\left(v_{h}-u_{h}\right), & \forall v_{h} \in \Lambda_{h} .\end{cases}
$$

We now assume that there exist two sub-spaces $W_{h}$ and $Z_{h}$ of $X_{h}$ and $M_{h}$ respectively which further satisfy

$$
W_{h} \subset K_{h}, \quad Z_{h} \subset \Lambda_{h}
$$

and define the subspace $V_{h}$ of $X_{h}$ by

$$
V_{h}:=\left\{q_{h} \in X_{h} ; \quad b\left(q_{h}, v_{h}\right)=0, \forall v_{h} \in Z_{h}\right\} .
$$

We also assume that both the following conforming hypothesis

$$
V_{h} \subset V
$$

and the uniform discrete inf-sup condition

$$
\exists \beta_{*}>0, \quad \sup _{q_{h} \in W_{h}} \frac{b\left(q_{h}, v_{h}\right)}{\left|q_{h}\right|_{X}} \geq \beta_{*}\left|v_{h}\right|_{M}, \quad \forall v_{h} \in M_{h},
$$

are satisfied. Observe that the conforming condition on $V_{h}$ ensures that the bilinear form $a$ is uniformly coercive on $V_{h}$. Theorem 2.3 and Lemma 2.2 apply in the discrete context too, and readily give that problem (18) has one and only one solution uniformly bounded in $h$.

For formulations where $K=X$ and $a$ is coercive on all of $X$, the well-posedness of the discrete problem and the convergence of its solution are obtained simply through the usual Brezzi's condition (20) with $W_{h}=X_{h}[2,10,18]$.

Theorem 2.6. Under hypotheses $(14,19)$ and (20), problem (18) has a unique solution $\left(p_{h}, u_{h}\right) \in K_{h} \times \Lambda_{h}$ satisfying the following estimate

$$
\left|p_{h}\right|_{X}+\left|u_{h}\right|_{M} \leq C\left(|L|_{X^{\prime}}+|\chi|_{M^{\prime}}\right)
$$

where the constant $C$ is independent of $h$ and remains bounded when $M_{a}, M_{b}, 1 / \alpha$ and $1 / \beta_{*}$ are varying on bounded subsets. 
Our aim now is to prove that $\left(p_{h}, u_{h}\right)$ converges to $(p, u)$ and to obtain an estimate of the errors $\left|p-p_{h}\right|_{X}$ and $\left|u-u_{h}\right|_{M}$. The linear case, corresponding to $K=X$ and $\Lambda=M$, is well-known [5]. When the first inequality reduces to an equation, that is, $K=X, \Lambda$ is a cone whose vertex is $0_{M}$ and the coercivity of $a$ holds on the whole space $X$, an error estimate and a convergence result can be found in [14,15]. The extension to the above general setting will be mainly obtained by coupling the techniques used for the two previous cases.

The following lemma establishes the key point at the basis of the convergence results and error estimates.

Lemma 2.7. Let $q_{h} \in K_{h}, q \in K, v_{h} \in \Lambda_{h}$ and $v \in \Lambda$. The following inequality holds

$$
\begin{aligned}
a\left(q_{h}-p_{h}, q_{h}-p_{h}\right) \leq A_{1}\left(q_{h}\right)+A_{2}(q)+ & B_{1}\left(v_{h}\right)+B_{2}(v) \\
& +a\left(q_{h}-p, q_{h}-p_{h}\right)+b\left(q_{h}-p, u_{h}-u\right)+b\left(p-p_{h}, u-v_{h}\right)
\end{aligned}
$$

where

$$
\begin{aligned}
A_{1}\left(q_{h}\right) & =a\left(p, q_{h}-p\right)+b\left(q_{h}-p, u\right)-L\left(q_{h}-p\right), \\
A_{2}(q) & =a\left(p, q-p_{h}\right)+b\left(q-p_{h}, u\right)-L\left(q-p_{h}\right), \\
B_{1}\left(v_{h}\right) & =b\left(p, u-v_{h}\right)-\chi\left(u-v_{h}\right), \\
B_{2}(v) & =b\left(p, u_{h}-v\right)-\chi\left(u_{h}-v\right) .
\end{aligned}
$$

Proof. We start from the following identity

$$
a\left(q_{h}-p_{h}, q_{h}-p_{h}\right)=a\left(q_{h}-p, q_{h}-p_{h}\right)+a\left(p-p_{h}, q_{h}-p_{h}\right)
$$

and develop the second term on the right hand side as follows

$$
a\left(p-p_{h}, q_{h}-p_{h}\right)=a\left(p-p_{h}, q_{h}-p\right)+a(p, p)+a\left(p_{h}, p_{h}\right)-a\left(p, p_{h}\right)-a\left(p_{h}, p\right) .
$$

Since $(p, u)$ and $\left(p_{h}, u_{h}\right)$ are the respective solutions to (1) and (18), we can write

$$
\begin{aligned}
a(p, p) & \leq L(p-q)+\chi(v-u)+a(p, q)+b(q, u)-b(p, v), \\
a\left(p_{h}, p_{h}\right) & \leq L\left(p_{h}-q_{h}\right)+\chi\left(v_{h}-u_{h}\right)+a\left(p_{h}, q_{h}\right)+b\left(q_{h}, u_{h}\right)-b\left(p_{h}, v_{h}\right) .
\end{aligned}
$$

Inequality (21) is then obtained by adequately gathering all the involved terms.

Remark 2.8. It is worth mentioning that when $K=X($ resp. $\Lambda=M)$ then $A_{1}(\cdot)=0\left(\right.$ resp. $\left.B_{1}(\cdot)=0\right)$. In some meaning, $A_{1}(\cdot)$ and $B_{1}(\cdot)$ give an estimate of the consistency error coming from the treatment of the nonlinearity in the problem. If $K_{h} \subset K$ (resp. $\left.\Lambda_{h} \subset \Lambda\right), \inf _{q \in K} A_{2}(q)=0$ (resp. $\left.\inf _{v \in \Lambda} B_{2}(v)=0\right)$ (simply take $q=p_{h}\left(\right.$ resp. $\left.\left.v=u_{h}\right)\right)$. Therefore, $A_{2}(\cdot)$ and $B_{2}(\cdot)$ yield the basic estimates for the consistency error coming from a non conforming approximation of either $K$ or $\Lambda$. Actually, this kind of estimate is an extension to the mixed formulations of the well-known Falk's lemma for usual variational inequalities [13].

Thanks to inf-sup condition (20), the following set

$$
V_{h}(\chi)=\left\{q_{h} \in K_{h} ; b\left(q_{h}, v_{h}\right)=\chi\left(v_{h}\right), \forall v_{h} \in Z_{h}\right\}
$$

is not empty. It will play a role as important as that of the similar subspace in the continuous problem.

Moreover, we suppose that the following inclusions, actually a kind of conforming hypotheses,

$$
W_{h} \subset W \text { and } Z_{h} \subset Z \text {, }
$$

are fulfilled. 
In all this section, $C$ denotes various constants, whose values may change from place to place, depending only and continuously on the parameters $M_{a}, M_{b}, 1 / \alpha$ and $1 / \beta_{*}$.

Lemma 2.9. Under the general assumption of Theorem 2.6, and moreover if the conforming conditions (22) are fulfilled, the following estimates hold

$$
\begin{aligned}
& \left|u-u_{h}\right|_{M} \leq C\left(\left|p-p_{h}\right|_{X}+\left|u-v_{h}\right|_{M}\right) \\
& \left|p-p_{h}\right|_{X}^{2} \leq C\left(A_{1}\left(q_{h}\right)+A_{2}(q)+B_{1}\left(v_{h}\right)+B_{2}(v)+\left|p-q_{h}\right|_{X}^{2}+\left|u-v_{h}\right|_{M}^{2}\right)
\end{aligned}
$$

for all $q_{h} \in V_{h}(\chi)$ and $v_{h} \in \Lambda_{h}$

Proof. We first use inf-sup condition (20) to obtain

$$
\beta_{*}\left|v_{h}-u_{h}\right|_{M} \leq \sup _{q_{h} \in W_{h}} \frac{b\left(q_{h}, v_{h}-u_{h}\right)}{\left|q_{h}\right|_{X}} .
$$

Since $W_{h}$ is a subspace and $W_{h} \subset W$, one can write that

$$
\begin{aligned}
b\left(q_{h}, v_{h}-u_{h}\right) & =b\left(q_{h}, v_{h}\right)+a\left(p_{h}, q_{h}\right)-L\left(q_{h}\right) \\
& =b\left(q_{h}, v_{h}-u\right)+a\left(p_{h}-p, q_{h}\right) .
\end{aligned}
$$

The triangle inequality directly yields estimate (23).

Next, taking $q_{h}$ in $V_{h}(\chi)$ and making use of the coercivity on $V_{h}$, we get the following bound from inequality (21)

$$
\begin{aligned}
\alpha\left|q_{h}-p_{h}\right|_{X}^{2} \leq A_{1}\left(q_{h}\right)+A_{2}(q) & +B_{1}\left(v_{h}\right)+B_{2}(v) \\
& +M_{a}\left|q_{h}-p\right|_{X}\left|q_{h}-p_{h}\right|_{X}+M_{b}\left(\left|q_{h}-p\right|_{X}\left|u_{h}-u\right|_{M}+\left|p-p_{h}\right|_{X}\left|u-v_{h}\right|_{M}\right) .
\end{aligned}
$$

The end of the proof is then obtained by a succession of straightforward bounds involving (23), the triangle inequality and an adequate use of the usual Young inequality

$$
a b \leq\left(a^{2} \varepsilon+b^{2} / \varepsilon\right) / 2, \text { for all } a, b \geq 0 \text { and } \varepsilon>0 .
$$

The next ingredient is to avoid the restriction on the approximating function $q_{h}$ to belong to $V_{h}(\chi)$.

Lemma 2.10. Under the general assumptions of Theorem 2.6, and moreover if conforming conditions (22) are fulfilled, there exists a map $T_{h}$ from $K_{h}$ into $V_{h}(\chi)$ such that

$$
\left\{\begin{array}{l}
\left|p-T_{h} r_{h}\right|_{X} \leq C\left|p-r_{h}\right|_{X}, \\
A_{1}\left(T_{h} r_{h}\right)=A_{1}\left(r_{h}\right),
\end{array} \quad \forall r_{h} \in K_{h}\right.
$$

Proof. It is based on an adaptation of a technique used for the linear case [5]. Let $r_{h}$ be any element of $K_{h}$. Discrete inf-sup condition (20) implies that there exists an element $w_{h} \in W_{h}$, which furthermore can be uniquely characterized, such that

$$
b\left(w_{h}, v_{h}\right)=b\left(p-r_{h}, v_{h}\right), \quad \forall v_{h} \in M_{h},
$$

which further satisfies the following bound [5]

$$
\left|w_{h}\right|_{X} \leq \beta_{*}^{-1} \sup _{v_{h} \in M_{h}} b\left(p-r_{h}, v_{h}\right) /\left|v_{h}\right|_{M} \leq \beta_{*}^{-1} M_{b}\left|p-r_{h}\right|_{X}
$$


We now use (10) to get that $T_{h} r_{h}:=r_{h}+w_{h}$ is in $K_{h}$. Indeed, we use (11) first to get that $b\left(p, v_{h}\right)=\chi\left(v_{h}\right)$ for all $v_{h} \in Z_{h}$ and next that $T_{h} r_{h}$ is in $V_{h}(\chi)$. The triangle inequality then gives

$$
\left|p-T_{h} r_{h}\right|_{X} \leq\left|p-r_{h}\right|_{X}+\left|w_{h}\right|_{X} \leq\left(1+\frac{M_{b}}{\beta_{*}}\right)\left|p-r_{h}\right|_{X} .
$$

Since $W_{h} \subset W$, the second point is a direct consequence of Lemma 2.1.

Theorem 2.11. Under hypotheses (14, 19, 20) and (22), if $(p, u),\left(p_{h}, u_{h}\right)$ are solutions to (1) and to (18) respectively, then the following error estimates hold

$$
\begin{gathered}
\left|p-p_{h}\right|_{X}^{2} \leq C\left(\inf _{q_{h} \in K_{h}}\left\{\left|p-q_{h}\right|_{X}^{2}+A_{1}\left(q_{h}\right)\right\}+\inf _{q \in K} A_{2}(q)\right. \\
\left.+\inf _{v_{h} \in \Lambda_{h}}\left\{\left|u-v_{h}\right|_{M}^{2}+B_{1}\left(v_{h}\right)\right\}+\inf _{v \in \Lambda} B_{2}(v)\right) \\
\left|u-u_{h}\right|_{M}^{2} \leq C\left(\left|p-p_{h}\right|_{X}^{2}+\inf _{v_{h} \in \Lambda_{h}}\left|u-v_{h}\right|_{M}^{2}\right) .
\end{gathered}
$$

The terms $A_{1}(\cdot), A_{2}(\cdot), B_{1}(\cdot)$ and $B_{2}(\cdot)$ are defined in Lemma 2.7.

Proof. It is a direct consequence of estimates $(23,24)$ and (25).

Remark 2.12. It is important to note that, when the bilinear form $a$ is coercive on the whole space $X$, Lemma 2.10 is no longer required to establish (27) and (28) since estimate (24) then holds for $q_{h}$ in $K_{h}$, not only when $q_{h} \in V_{h}(\chi)$. This is the frame of the estimates given in [2,10,18]. Unfortunately, such a property does not hold in a mixed formulation involving both the displacements and the stresses and explicitly enforcing the equilibrium conditions.

Estimates $(27)$ and $(28)$ can be used to verify that the discrete solution $\left(p_{h}, u_{h}\right)$ converges to $(p, u)$ strongly, as this will be established in what follows. They also yield an estimate of the rate of this convergence for the mixed finite element approximation of the above unilateral boundary-value problems.

Definition 2.13. The family of subsets $\left\{\mathcal{C}_{h}\right\}_{h>0}$ of $X$ is said to approach $\mathcal{C} \subset X$ in the sense of Mosco [20] if the following conditions hold

$\forall v \in \mathcal{C}$, there exists $\left\{v_{h}\right\}_{h>0}$ such that $v_{h} \in \mathcal{C}_{h}, \forall h>0$ and $\lim _{h \rightarrow 0} v_{h}=v$ strongly in $X$, if $v_{h} \in \mathcal{C}_{h}, \forall h>0$ and $\lim _{h \rightarrow 0} v_{h}=v$ weakly in $X$ then $v \in \mathcal{C}$.

We are now in position to prove the following convergence theorem.

Theorem 2.14. Assume that $\left\{K_{h}\right\}_{h>0}$ and $\left\{\Lambda_{h}\right\}_{h>0}$ approach $K$ and $\Lambda$ respectively in the sense of Mosco. Then, under the general conditions of Theorem 2.11, $\left(p_{h}, u_{h}\right)$ converges to $(p, u)$ strongly in $X \times M$.

Proof. First, Lemma 2.2 ensures that there exists a constant $C$ independent of $h>0$ such that

$$
\left|p_{h}\right|_{X}+\left|u_{h}\right|_{M} \leq C
$$

Hence, there exists a sub-sequence still denoted by $\left\{\left(p_{h}, u_{h}\right)\right\}_{h>0}$ and an element $\left(p_{*}, u_{*}\right) \in X \times M$ such that

$$
\lim _{h \rightarrow 0} p_{h}=p_{*} \text { weakly in } X, \text { and } \lim _{h \rightarrow 0} u_{h}=u_{*} \text { weakly in } M \text {. }
$$


Since $K_{h}$ and $\Lambda_{h}$ approach $K$ and $\Lambda$ respectively, $\left(p_{*}, u_{*}\right)$ belongs to $K \times \Lambda$. In view of (29), by possibly passing once again to a sub-sequence, we can indeed assume that

$$
\lim _{h \rightarrow 0} a\left(p_{h}, p_{h}\right)=a_{*} \text { and } \lim _{h \rightarrow 0} b\left(p_{h}, u_{h}\right)=b_{*} .
$$

Let us show now that $\left(p_{*}, u_{*}\right)$ gives a solution to $(18)$. Let $(q, v)$ be fixed in $K \times \Lambda$. The approximation property yields that there exists a sequence $\left\{\left(q_{h}, v_{h}\right)\right\}_{h>0}$ in $K_{h} \times \Lambda_{h}$ such that

$$
\lim _{h \rightarrow 0} q_{h}=q \text { strongly in } X \text { and } \lim _{h \rightarrow 0} v_{h}=v \text { strongly in } M .
$$

Since $\left(p_{h}, u_{h}\right)$ is a solution to (18), they verify

$$
\begin{aligned}
a\left(p_{h}, p_{h}-q_{h}\right)+b\left(p_{h}-q_{h}, u_{h}\right) & \leq L\left(p_{h}-q_{h}\right), \forall q_{h} \in K_{h}, \\
b\left(p_{h}, v_{h}-u_{h}\right) & \leq \chi\left(v_{h}-u_{h}\right), \forall v_{h} \in \Lambda_{h} .
\end{aligned}
$$

Since the bilinear form $a$ is non negative on $X$, letting $h \rightarrow 0$ in (31), we first get that $a\left(p_{*}, p_{*}\right) \leq a_{*}$ and, next, that

$$
a\left(p_{*}, p_{*}-q\right)+b_{*}-b\left(q, u_{*}\right) \leq L\left(p_{*}-q\right) .
$$

The same procedure applies to $(32)$ yielding

$$
b\left(p_{*}, v\right)-\chi\left(v-u_{*}\right) \leq b_{*} .
$$

Taking $v=u_{*}$ in (34), we obtain that $b\left(p_{*}, u_{*}\right) \leq b_{*}$. Then, in view of (33), this inequality gives

$$
a\left(p_{*}, p_{*}-q\right)+b\left(p_{*}-q, u_{*}\right) \leq L\left(p_{*}-q\right), \forall q \in K .
$$

In the same way, we take $q=p_{*}$ in (33) to get that

$$
b_{*} \leq b\left(p_{*}, u_{*}\right) .
$$

Inequality (34) then yields

$$
b\left(p_{*}, v-u_{*}\right) \leq \chi\left(v-u_{*}\right), \quad \forall v \in \Lambda .
$$

Thus, $\left(p_{*}, u_{*}\right)$ is a solution to (1). From the uniqueness of this solution, we get that the whole sequence $\left\{\left(p_{h}, u_{h}\right)\right\}_{h>0}$ converges weakly to $(p, u)$ as $h$ tends to 0 . In order to prove that it indeed converges strongly in $X \times M$, we consider $\pi_{K_{h}} p \in K_{h}$ and $\pi_{\Lambda_{h}} u \in \Lambda_{h}$ the respective projection of $p$ on $K_{h}$ and of $u$ on $\Lambda_{h}$. From the assumed convergence of $K_{h}$ to $K$ and of $\Lambda_{h}$ to $\Lambda$ in the sense of Mosco, they converge to $p$ and $u$ strongly respectively in $X$ and $M$. Taking $q=p, v=u, q_{h}=\pi_{K_{h}} p$ and $v_{h}=\pi_{\Lambda_{h}} u$ in (27) and (28) respectively, readily completes the proof.

\section{EXTENSION TO INEQUALITIES INVOLVING A THIRD BILINEAR FORM}

\subsection{The continuous problem}

Now, we intent to extend the study done for problem (1) to the following type of mixed formulations

$$
\begin{cases}(p, u) \in K \times \Lambda, & \\ a(p, q-p)+b(q-p, u) \geq L(q-p), & \forall q \in K, \\ b(p, v-u)-c(u, v-u) \leq \chi(v-u), & \forall v \in \Lambda,\end{cases}
$$


which involves a third bilinear form $c$ on $M \times M$ assumed to be symmetric, i.e.,

$$
c(u, v)=c(v, u), \forall u, v \in M
$$

continuous, i.e., satisfying

$$
\exists M_{c}>0,|c(u, v)| \leq M_{c}|u|_{M}|v|_{M}, \forall u, v \in M,
$$

and non negative, i.e.,

$$
c(v, v) \geq 0 \text { for all } v \in M .
$$

Note that the form $c$ is not assumed to be coercive nor even definite.

Remark 3.1. If the form $a$ is symmetric, problem (35) is equivalent to the following saddle point problem $(c f .[12])$

$$
\left\{\begin{array}{l}
(p, u) \in K \times \Lambda, \\
\mathcal{L}(p, v) \leq \mathcal{L}(p, u) \leq \mathcal{L}(q, u), \quad \forall(q, v) \in K \times \Lambda,
\end{array}\right.
$$

where the Lagrangian $\mathcal{L}$ is given by

$$
\mathcal{L}(q, v)=\frac{1}{2} a(q, q)-L(q)-\frac{1}{2} c(v, v)+b(q, v)-\chi(v) .
$$

In the case where $K=X$ and $\Lambda=M$, problem (35) reduces to the following linear one

$$
\begin{cases}(p, u) \in X \times M & \\ a(p, q)+b(q, u)=L(q), & \forall q \in X \\ b(p, v)-c(u, v)=\chi(v), & \forall v \in M\end{cases}
$$

The study of this problem has been tackled in [5] where the existence of a solution has been established using perturbation techniques. Several examples of mixed formulations which can be obtained as special instances of this general setting can be found therein.

Actually, the third bilinear form $c$ introduces a strong coupling between the two unknowns $p$ and $u$ also at the level of the second (in)equality. This makes it more difficult to exploit the restricted coercivity of $a$ on $V$ only. For the linear case, it is the complete characterization of the continuity of the inverse operator by its boundedness on the unit ball and the superposition principle which permit the extension of the results obtained for the case $c=0$ to problem (39) [5]. Unfortunately, these techniques cannot be adapted for inequalities.

From now on in this section, we focus on the following example which is related to a stabilization procedure for the mixed formulation of the Signorini problem (3)

$$
\left\{\begin{array}{l}
\boldsymbol{p}^{\varepsilon} \in \boldsymbol{K}, \quad \forall \boldsymbol{q} \in \boldsymbol{K} \\
\int_{\Omega} \boldsymbol{A}^{-1} \boldsymbol{p}^{\varepsilon} \cdot\left(\boldsymbol{q}-\boldsymbol{p}^{\varepsilon}\right) \mathrm{d} \Omega+\frac{1}{\varepsilon} \int_{\Omega} \boldsymbol{\nabla} \cdot \boldsymbol{p}^{\varepsilon} \boldsymbol{\nabla} \cdot\left(\boldsymbol{q}-\boldsymbol{p}^{\varepsilon}\right) \mathrm{d} \Omega \geq-\frac{1}{\varepsilon} \int_{\Omega} f \boldsymbol{\nabla} \cdot\left(\boldsymbol{q}-\boldsymbol{p}^{\varepsilon}\right) \mathrm{d} \Omega
\end{array}\right.
$$

where $\boldsymbol{K}$ is the convex cone of $H(\operatorname{div} ; \Omega)$ defined in (4) and $\varepsilon$ is a penalty parameter destined to tend to zero. Setting $u^{\varepsilon}=\left(\boldsymbol{\nabla} \cdot \boldsymbol{p}^{\varepsilon}+f\right) / \varepsilon$ reduces problem (40) to the extension of problem (5) involving as third form

$$
c(u, v):=\varepsilon \int_{\Omega} u v \mathrm{~d} \Omega
$$

Observe that in this case $c$ is moreover definite and that $M_{c}$ can be assumed to be as small as necessary. 
So in view of the previous example, we can assume that the bilinear forms involved in problem (35) satisfy the following condition in addition to those already stated in the previous section

$$
a(q, q)+c(v, v)=0 \text { if and only if either } q=0 \text { or } v=0 .
$$

Observe that condition (41) will be needed to establish the uniqueness of the solution only.

Now, we try to establish the existence and the uniqueness of a solution to (35). They are obtained similarly to the case $c=0$. The main step is to obtain an a priori estimate like the one established in Lemma 2.2. Indeed, the former is based on the latter and an adaptation of the treatment of the linear case (39) done in [5].

Lemma 3.2. As in Lemma 2.2, assume that the bilinear forms a and $b$ as well as the linear forms $L$ and $\chi$ are continuous and that conditions $(12,13)$ and $(14)$ hold and moreover that the bilinear form $c$ satisfies the above conditions $(36,37)$ and (38), then any solution $(p, u)$ to problem (35) fulfills the following a priori estimate

$$
|p|_{X}+|u|_{M} \leq C\left(|L|_{X^{\prime}}+|\chi|_{M^{\prime}}\right),
$$

where $C$ is a positive constant which remains bounded if $M_{a}, M_{b}, M_{c}, 1 / \alpha$ and $1 / \beta$ are varying in a bounded set.

Proof. Using the symmetry of $c$, we first get that

$$
|c(u, v)| \leq c(u, u)^{1 / 2} c(v, v)^{1 / 2} \leq M_{c}^{1 / 2} c(u, u)^{1 / 2}|v|_{M}, \quad \forall u, v \in M .
$$

Hence, according to Lemma 2.2, we can conclude that

$$
|p|_{X}+|u|_{M} \leq C\left(|L|_{X^{\prime}}+|\chi|_{M^{\prime}}+M_{c}^{1 / 2} c(u, u)^{1 / 2}\right)
$$

where $C$ is the constant involved in this lemma.

To deal with the term $c(u, u)^{1 / 2}$, we take $q=0$ and $v=0$ in (35), hence getting

$$
a(p, p)+c(u, u) \leq L(p)-\chi(u),
$$

and next

The above estimates imply that

$$
c(u, u) \leq|L|_{X^{\prime}}|p|_{X}+|\chi|_{M^{\prime}}|u|_{M} .
$$

$$
c(u, u) \leq C\left(|L|_{X^{\prime}}+|\chi|_{M^{\prime}}\right)\left(|L|_{X^{\prime}}+|\chi|_{M^{\prime}}+c(u, u)^{1 / 2}\right)
$$

with a constant $C$ owning the property stated above. Since $c(u, u) \geq 0$, an elementary proof yields that there exists another constant $C$ with the same property such that

$$
c(u, u)^{1 / 2} \leq C\left(|L|_{X^{\prime}}+|\chi|_{M^{\prime}}\right) .
$$

In view of the already obtained bounds, this completes the proof of the lemma.

We now state the main theorem in this part.

Theorem 3.3. Under the general hypotheses of Lemma 3.2, problem (35) admits a solution satisfying

$$
|p|_{X}+|u|_{M} \leq C\left(|L|_{X^{\prime}}+|\chi|_{M^{\prime}}\right),
$$

with a constant $C$ which remains bounded whenever $M_{a}, M_{b}, M_{c}, 1 / \alpha$ and $1 / \beta$ are varying on a bounded set. If moreover the forms $a$ and $c$ are such that condition (41) holds, this solution is unique. 
Proof. The existence of a solution can be obtained exactly as for the case $c=0$. Likewise, to prove the uniqueness, we first get

$$
\begin{gathered}
a\left(p_{1}-p_{2}, p_{1}-p_{2}\right)+b\left(p_{1}-p_{2}, u_{1}-u_{2}\right) \leq 0 \\
b\left(p_{2}-p_{1}, u_{1}-u_{2}\right)+c\left(u_{1}-u_{2}, u_{1}-u_{2}\right) \leq 0
\end{gathered}
$$

The fact that $a$ and $c$ are non negative implies

$$
b\left(p_{1}-p_{2}, u_{1}-u_{2}\right)=0 .
$$

Hence, we deduce that

$$
a\left(p_{1}-p_{2}, p_{1}-p_{2}\right)+c\left(u_{1}-u_{2}, u_{1}-u_{2}\right)=0 .
$$

It is not clear that $p_{1}-p_{2}$ belongs to $V$. So coercivity of $a$ on $V$ is of no use now. Instead, it is condition (41) which yields the alternative $p_{1}-p_{2}=0$ or $u_{1}-u_{2}=0$. We discuss each of the two occurrences separately.

If $p_{1}=p_{2}$, the uniqueness of component $u$ results from the inf-sup condition as in the proof of Theorem 2.3. When $u_{1}=u_{2}$, moving $c(u, v-u)$ to the right-hand side and using the uniqueness already obtained for the case $c=0$ directly establishes that $p_{1}=p_{2}$ too.

Remark 3.4. As mentioned above, when the bilinear form $a$ is symmetric, problem (35) is equivalent to a saddle point problem. Thus, if $\left(p_{1}, u_{1}\right)$ and $\left(p_{2}, u_{2}\right)$ are two saddle points for the Lagrangian $\mathcal{L}$, then $\left(p_{1}, u_{2}\right)$ and $\left(p_{2}, u_{1}\right)$ also give two other saddle points for $\mathcal{L}[12]$. As a result, $p_{1}-p_{2}$ belongs to $V$. This provides the uniqueness without supposing condition (41) to hold. For this reason, we are inclined to think that uniqueness occurs in the nonsymmetric case too, without appealing to property (41).

\subsection{The discrete problem and error estimates}

Keeping the general procedure and the assumptions described in Section 2, the discrete version of problem (35) can be stated as follows

$$
\left\{\begin{array}{l}
\left(p_{h}, u_{h}\right) \in K_{h} \times \Lambda_{h} \\
a\left(p_{h}, q_{h}-p_{h}\right)+b\left(q_{h}-p_{h}, u_{h}\right) \geq L\left(q_{h}-p_{h}\right), \quad \forall q_{h} \in K_{h}, \\
b\left(p_{h}, v_{h}-u_{h}\right)-c\left(u_{h}, v_{h}-u_{h}\right) \leq \chi\left(v_{h}-u_{h}\right), \quad \forall v_{h} \in \Lambda_{h} .
\end{array}\right.
$$

This approximation of problem (35) can be studied along almost the same lines as that already done for the case $c=0$. First, the following theorem is a simple instance of the general conclusion stated in Theorem 3.3.

Theorem 3.5. Under the general assumptions of Lemma 3.2 and Theorem 3.3 and hypotheses (19) and (20), problem (45) admits at least one solution $\left(p_{h}, u_{h}\right)$ satisfying

$$
\left|p_{h}\right|_{X}+\left|u_{h}\right|_{M} \leq C\left(|L|_{X^{\prime}}+|\chi|_{M^{\prime}}\right)
$$

where $C$ is a positive constant independent of $h$ and remains bounded on bounded subsets of $M_{a}, M_{b}, M_{c}, 1 / \alpha, 1 / \beta_{*}$. Moreover, if condition (41) is fulfilled, this solution is unique.

In all of this section, $(p, u)$ and $\left(p_{h}, u_{h}\right)$ will now denote the respective solutions to problems (35) and (45). We now prove the counterpart of Lemma 2.7 .

Lemma 3.6. For any $q_{h} \in K_{h}, q \in K, v_{h} \in \Lambda_{h}$ and $v \in \Lambda$, the following inequality holds

$$
\begin{aligned}
a\left(q_{h}-p_{h}, q_{h}-p_{h}\right)+c & \left(u_{h}-u, u_{h}-u\right) \leq A_{1}\left(q_{h}\right)+A_{2}(q)+B_{1}\left(v_{h}\right)+B_{2}(v) \\
& +a\left(q_{h}-p, q_{h}-p_{h}\right)+c\left(u_{h}-u, v_{h}-u\right)+b\left(p-p_{h}, v_{h}-u\right)+b\left(q_{h}-p, u_{h}-u\right)
\end{aligned}
$$


where

$$
\begin{aligned}
A_{1}\left(q_{h}\right) & =a\left(p, q_{h}-p\right)+b\left(q_{h}-p, u\right)-L\left(q_{h}-p\right), \\
A_{2}(q) & =a\left(p, q-p_{h}\right)+b\left(q-p_{h}, u\right)-L\left(q-p_{h}\right), \\
B_{1}\left(v_{h}\right) & =b\left(p, u-v_{h}\right)-c\left(u, u-v_{h}\right)-\chi\left(u-v_{h}\right), \\
B_{2}(v) & =b\left(p, u_{h}-v\right)-c\left(u, u_{h}-v\right)-\chi\left(u_{h}-v\right) .
\end{aligned}
$$

Proof. Proceeding exactly as for the proof of Lemma 2.7, we obtain

$$
\begin{aligned}
a\left(q_{h}-p_{h}, q_{h}-p_{h}\right) \leq A_{1}\left(q_{h}\right)+A_{2}(q)+B_{1}\left(v_{h}\right) & +B_{2}(v)+a\left(q_{h}-p, q_{h}-p_{h}\right) \\
& +c\left(u_{h}-u, v_{h}-u_{h}\right)+b\left(p-p_{h}, v_{h}-u\right)+b\left(q_{h}-p, u_{h}-u\right) .
\end{aligned}
$$

Writing $c\left(u_{h}-u, v_{h}-u_{h}\right)$ in the form $c\left(u_{h}-u, v_{h}-u\right)-c\left(u_{h}-u, u_{h}-u\right)$ directly leads to (46).

The strong coupling of $p_{h}$ and $u_{h}$ in the second inequality of (35) makes the analysis of the present discrete solution much more involved since now the set $V_{h}(\chi)$, introduced in the previous section, depends not only on $\chi$ but also on $u_{h}$

$$
V_{h}\left(\chi, u_{h}\right):=\left\{q_{h} \in K_{h} ; b\left(q_{h}, v_{h}\right)=c\left(u_{h}, v_{h}\right)+\chi\left(v_{h}\right), \forall v_{h} \in Z_{h}\right\} .
$$

The proof of the following lemma is very similar to that of Lemma 2.9 and will not be repeated here.

Lemma 3.7. Under the general assumption of Theorem 3.5, and if moreover the conforming conditions (22) are fulfilled, the following estimates hold

$$
\begin{gathered}
\left|u-u_{h}\right|_{M} \leq C\left(\left|p-p_{h}\right|_{X}+\left|u-v_{h}\right|_{M}\right), \\
\left.\left|p-p_{h}\right|_{X}^{2}+c\left(u-u_{h}, u-u_{h}\right) \leq C\left(A_{1}\left(q_{h}\right)+A_{2}(q)+B_{1}\left(v_{h}\right)+B_{2}(v)+\left|p-q_{h}\right|_{X}^{2}+\left|u-v_{h}\right|_{M}^{2}\right)\right)
\end{gathered}
$$

for all $q_{h} \in V_{h}\left(\chi, u_{h}\right)$ and all $v_{h} \in \Lambda_{h}$.

We come now to the most particular feature of the problem involving the third bilinear form.

Lemma 3.8. Under the general assumptions of Theorem 3.5, and if moreover conforming conditions (22) are fulfilled, there exists a map $T_{h}$ from $K_{h}$ into $V_{h}\left(\chi, u_{h}\right)$ such that

$$
\begin{gathered}
\left|p-T_{h} r_{h}\right|_{X} \leq C\left|p-r_{h}\right|_{X}+\frac{M_{c}^{1 / 2}}{\beta_{*}} c\left(u-u_{h}, u-u_{h}\right)^{1 / 2}, \quad \forall r_{h} \in K_{h}, \\
A_{1}\left(T_{h} r_{h}\right)=A_{1}\left(r_{h}\right), \quad \forall r_{h} \in K_{h} .
\end{gathered}
$$

Proof. We now define $w_{h} \in W_{h}$ by

$$
b\left(w_{h}, v_{h}\right)=b\left(p-r_{h}, v_{h}\right)-c\left(u-u_{h}, v_{h}\right), \quad \forall v_{h} \in M_{h} .
$$

Using the fact that $Z_{h} \subset Z$ and Lemma 2.1 and defining $T_{h} r_{h}:=r_{h}+w_{h}$, we get

$$
b\left(T_{h} r_{h}, v_{h}\right)=\chi\left(v_{h}\right)+c\left(u_{h}, v_{h}\right), \quad \forall v_{h} \in Z_{h},
$$

that is, $T_{h} r_{h} \in V_{h}\left(\chi, u_{h}\right)$. The bound on $\left|p-T_{h} r_{h}\right|_{X}$ results from the following ones

$$
c\left(u-u_{h}, v_{h}\right) \leq c\left(u-u_{h}, u-u_{h}\right)^{1 / 2} c\left(v_{h}, v_{h}\right)^{1 / 2} \leq M_{c}^{1 / 2} c\left(u-u_{h}, u-u_{h}\right)^{1 / 2}\left|v_{h}\right|_{M} .
$$

The rest of the proof is straightforward.

In view of the results stated in the previous lemmas, we can readily prove the following theorem. 
Theorem 3.9. Let $(p, u)$ and $\left(p_{h}, u_{h}\right)$ be the solutions to (35) and (45) respectively. Under general conditions (19, 20, 22, 36, 37) and (38), there exists a constant $M_{0}>0$ depending only on $M_{a}, M_{b}, 1 / \alpha$ and $1 / \beta_{*}$ such that if $M_{c}<M_{0}$, then the following estimates hold

$$
\begin{aligned}
\left|p-p_{h}\right|_{X}^{2} \leq & C_{1}\left(\inf _{q_{h} \in K_{h}}\left\{\left|p-q_{h}\right|_{X}^{2}+A_{1}\left(q_{h}\right)\right\}+\inf _{q \in K} A_{2}(q)\right. \\
& \left.+\inf _{v_{h} \in \Lambda_{h}}\left\{\left|u-v_{h}\right|_{M}^{2}+B_{1}\left(v_{h}\right)\right\}+\inf _{v \in \Lambda} B_{2}(v)\right), \\
\left|u-u_{h}\right|_{M}^{2} \leq & C_{2}\left(\left|p-p_{h}\right|_{X}^{2}+\inf _{v_{h} \in \Lambda_{h}}\left|u-v_{h}\right|_{M}^{2}\right),
\end{aligned}
$$

where $C_{1}, C_{2}$ are positive constants independent of $h$, and $A_{1}(\cdot), A_{2}(\cdot), B_{1}(\cdot)$ and $B_{2}(\cdot)$ being defined as in Lemma 3.6.

Proof. Let $r_{h}$ be any element in $K_{h}$. Taking $q_{h}=T_{h} r_{h}$ in (48) and using (50), we obtain

$$
\left|p-p_{h}\right|_{X}^{2}+c\left(u-u_{h}, u-u_{h}\right) \leq C\left(A_{1}\left(r_{h}\right)+A_{2}(q)+B_{1}\left(v_{h}\right)+B_{2}(v)+\left|p-T_{h} r_{h}\right|_{X}^{2}+\left|u-v_{h}\right|_{M}^{2}\right)
$$

From (49), we can get now

$$
\left|p-T_{h} r_{h}\right|_{X}^{2} \leq C\left|p-r_{h}\right|_{X}^{2}+2\left(M_{c} / \beta_{*}^{2}\right) c\left(u_{h}-u, u_{h}-u\right)
$$

Clearly, the rest of the proof can be obtained in a straightforward way.

Remark 3.10. In the same way as in Remark 2.12, when the bilinear form $a$ is coercive on the whole space $X$, the error estimates given in Theorem 3.9 can be established without using Lemma 3.8. Therefore, the condition on $M_{c}$ to be small enough in order for the estimate to hold becomes irrelevant. This is particularly the case for some mixed formulations of elasticity problems involving a nearly incompressible material (see [5] for the linear case and [3] for a boundary condition relative to a unilateral contact).

Similarly as in the proof of Theorem 2.14, we can show that the following convergence result holds.

Theorem 3.11. Assume that $\left\{K_{h}\right\}_{h>0}$ and $\left\{\Lambda_{h}\right\}_{h>0}$ approach $K$ and $\Lambda$ respectively in the sense of Mosco. Then, under the general conditions of the previous theorem, the solution $\left(p_{h}, u_{h}\right)$ to problem (45) converges strongly in $X \times M$ to the solution $(p, u)$ to problem (35).

Remark 3.12. If $K=X(\operatorname{resp} . \Lambda=M)$ then $A_{1}\left(q_{h}\right)=0$ (resp. $\left.B_{1}\left(v_{h}\right)=0\right)$. When $K_{h} \subset K\left(\right.$ resp. $\left.\Lambda_{h} \subset \Lambda\right)$, $\inf _{q \in K} A_{2}(q)=0\left(\operatorname{resp} \inf _{v \in \Lambda} B_{2}(v)=0\right)$.

\section{Application}

In this section, the results obtained in the context of the above general setting are applied to the study of the mixed finite element approximation of the Signorini problem. We assume that the $\Omega$ is a polygonal domain, and that $\mathcal{T}_{h}$ is a regular mesh [9] of $\Omega$ in triangles denoted in a generic way by $T$. We assume that $\mathcal{T}_{h}$ is compatible with the partitioning of $\partial \Omega$ in $\Gamma_{D}, \Gamma_{N}$ and $\Gamma_{C}$. As a result, the shape functions are taken in the Raviart-Thomas space of polynomials of the lowest degree (cf. [23])

$$
R T_{0}=\mathbb{P}_{0}^{2}+\mathbb{P}_{0} \boldsymbol{r}
$$


where $\boldsymbol{r}(x)$ is the radius vector of point $x \in \mathbb{R}^{2}$ and $\mathbb{P}_{0}$ is the space of constant functions. Defining the following finite element spaces

$$
\begin{aligned}
& \boldsymbol{X}_{h}=\left\{\boldsymbol{q}_{h} \in H(\operatorname{div} ; \Omega) ;\left.\quad \boldsymbol{q}_{h}\right|_{T} \in R T_{0}, \forall T \in \mathcal{T}_{h}\right\}, \\
& M_{h}=\left\{v_{h} \in L^{2}(\Omega) ;\left.v_{h}\right|_{T} \in \mathbb{P}_{0}, \forall T \in \mathcal{T}_{h}\right\},
\end{aligned}
$$

we can consider the following discrete approximation of the convex cone being involved in the mixed formulation of the Signorini problem

$$
\boldsymbol{K}_{h}=\left\{\boldsymbol{q}_{h} \in \boldsymbol{X}_{h} ; \quad \boldsymbol{q}_{h} \cdot \boldsymbol{n}=0 \text { on } \Gamma_{N}, \quad \boldsymbol{q}_{h} \cdot \boldsymbol{n} \geq 0 \text { on } \Gamma_{C}\right\} .
$$

The conditions on the boundary $\Gamma_{N}$ and $\Gamma_{C}$ are taken in the usual sense. Hence, we directly get the approximation of the mixed formulation (5) by the Raviart-Thomas finite elements of the lowest degree

$$
\begin{cases}\left(\boldsymbol{p}_{h}, u_{h}\right) \in \boldsymbol{K}_{h} \times M_{h}, & \\ a\left(\boldsymbol{p}_{h}, \boldsymbol{q}_{h}-\boldsymbol{p}_{h}\right)+b\left(\boldsymbol{q}_{h}-\boldsymbol{p}_{h}, u_{h}\right) \geq 0, & \forall \boldsymbol{q}_{h} \in \boldsymbol{K}_{h}, \\ b\left(\boldsymbol{p}_{h}, v_{h}\right)=-\left(f, v_{h}\right), & \forall v_{h} \in M_{h} .\end{cases}
$$

Defining

which is nothing else but

$$
\boldsymbol{X}_{0, h}:=\left\{\boldsymbol{q}_{h} \in \boldsymbol{X}_{h} ; \quad b\left(\boldsymbol{q}_{h}, v_{h}\right)=0, \forall v_{h} \in M_{h}\right\}
$$

$$
\boldsymbol{X}_{0, h}=\left\{\boldsymbol{q}_{h} \in \boldsymbol{X}_{h} ; \boldsymbol{\nabla} \cdot \boldsymbol{q}_{h}=0 \text { in } \Omega\right\},
$$

we observe that, as for the continuous case, the following properties

$$
\begin{gathered}
\exists \alpha>0: a\left(\boldsymbol{q}_{h}, \boldsymbol{q}_{h}\right) \geq \alpha\left\|\boldsymbol{q}_{h}\right\|_{H(\operatorname{div} ; \Omega)}^{2}, \quad \forall \boldsymbol{q}_{h} \in \boldsymbol{X}_{0, h}, \\
\exists \beta_{*}>0: \sup _{\boldsymbol{q}_{h} \in \boldsymbol{W}_{h}} \frac{b\left(\boldsymbol{q}_{h}, v_{h}\right)}{\left\|\boldsymbol{q}_{h}\right\|_{H(\operatorname{div} ; \Omega)}} \geq \beta_{*}\left\|v_{h}\right\|_{0, \Omega}, \quad \forall v_{h} \in M_{h},
\end{gathered}
$$

hold uniformly in $h$ (see [5]). We have denoted by

$$
\boldsymbol{W}_{h}:=\left\{\boldsymbol{q}_{h} \in \boldsymbol{X}_{h} ; \quad \boldsymbol{q}_{h} \cdot \boldsymbol{n}=0 \text { on } \Gamma_{C} \cup \Gamma_{N}\right\},
$$

that is, the intersection of the cones $\boldsymbol{K}_{h}$ and $-\boldsymbol{K}_{h}$. Theorem 2.6 yields that problem (51) has one and only one solution $\left(\boldsymbol{p}_{h}, u_{h}\right)$. In this section, we seek a bound for the error resulting from this approximation process.

Proposition 4.1. Recall that $\boldsymbol{p}$ is the solution to the continuous problem (5). The following error estimate holds

$$
\begin{aligned}
\left\|\boldsymbol{p}-\boldsymbol{p}_{h}\right\|_{H(\operatorname{div} ; \Omega)}^{2} & \leq C\left(\inf _{\boldsymbol{q}_{h} \in \boldsymbol{K}_{h}}\left\{\left\|\boldsymbol{p}-\boldsymbol{q}_{h}\right\|_{H(\operatorname{div} ; \Omega)}^{2}+\left\langle\left(\boldsymbol{q}_{h}-\boldsymbol{p}\right) \cdot \boldsymbol{n}, u\right\rangle_{1 / 2, \partial \Omega}\right\}+\inf _{v_{h} \in M_{h}}\left\|u-v_{h}\right\|_{0, \Omega}^{2}\right) \\
\left\|u-u_{h}\right\|_{0, \Omega} & \leq C\left(\left\|\boldsymbol{p}-\boldsymbol{p}_{h}\right\|_{H(\operatorname{div} ; \Omega)}+\inf _{v_{h} \in M_{h}}\left\|u-v_{h}\right\|_{0, \Omega}\right)
\end{aligned}
$$

where $\langle., .\rangle_{1 / 2, \partial \Omega}$ denotes the duality pairing between $H^{1 / 2}(\partial \Omega)$ and $H^{-1 / 2}(\partial \Omega)$ and $C$ is a positive constant independent of $h$.

Proof. Here, in the notation of problems (1) and (18), only the term $A_{1}\left(q_{h}\right)$ has to be considered since $\Lambda=M$, $K_{h} \subset K$ and $M_{h} \subset M$. It is given by

$$
A_{1}\left(q_{h}\right)=\int_{\Omega}\left(\boldsymbol{A}^{-1} \boldsymbol{p} \cdot\left(\boldsymbol{q}_{h}-\boldsymbol{p}\right)+u \boldsymbol{\nabla} \cdot\left(\boldsymbol{q}_{h}-\boldsymbol{p}\right)\right) \mathrm{d} \Omega .
$$


Since $\boldsymbol{q} \in \mathcal{D}\left(\Omega ; \mathbb{R}^{2}\right) \subset \boldsymbol{K}$, the first inequality gives that $u \in H^{1}(\Omega)$ and

$$
\nabla u=A^{-1} p
$$

The usual Green formula then shows that $A_{1}\left(q_{h}\right)$ is nothing else but

$$
A_{1}\left(q_{h}\right)=\left\langle\left(\boldsymbol{q}_{h}-\boldsymbol{p}\right) \cdot \boldsymbol{n}, u\right\rangle_{1 / 2, \partial \Omega} .
$$

The rest of the proof is a direct consequence of Theorem 2.11.

To prove that $\left(\boldsymbol{p}_{h}, u_{h}\right)$ converges to $(\boldsymbol{p}, u)$, we need to establish that $\boldsymbol{K}_{h}$ converges to $\boldsymbol{K}$ in the sense of Mosco. As $\boldsymbol{K}_{h} \subset \boldsymbol{K}$, it is enough to show that

$$
\forall \boldsymbol{q} \in \boldsymbol{K}, \lim _{h \rightarrow 0} \inf _{\boldsymbol{q}_{h} \in \boldsymbol{K}_{h}}\left\|\boldsymbol{q}-\boldsymbol{q}_{h}\right\|_{H(\operatorname{div} ; \Omega)}=0 .
$$

The first ingredient will be to prove the following density lemma.

Lemma 4.2. The convex cone $\mathcal{K}$ defined by

$$
\mathcal{K}=\left\{\boldsymbol{q} \in \mathcal{D}\left(\bar{\Omega} ; \mathbb{R}^{2}\right) ; \boldsymbol{q} \cdot \boldsymbol{n}=0 \text { on } \Gamma_{N} \text { and } \boldsymbol{q} \cdot \boldsymbol{n} \geq 0 \text { on } \Gamma_{C}\right\}
$$

is dense in $\boldsymbol{K}$.

Proof. The proof is by contradiction. We first use the well-known identification of $H(\operatorname{div} ; \Omega)$ to a closed subspace of $L^{2}\left(\Omega ; \mathbb{R}^{2}\right) \times L^{2}(\Omega)$ through the following injective mapping

$$
\boldsymbol{q} \longmapsto(\boldsymbol{q}, \boldsymbol{\nabla} \cdot \boldsymbol{q})
$$

Assume that there exists an element $\boldsymbol{p}_{0}$ in $\boldsymbol{K}$ not belonging to the closure $\overline{\mathcal{K}}$ of $\mathcal{K}$ in $H(\operatorname{div} ; \Omega)$. Since $\overline{\mathcal{K}}$ can be identified to a closed convex subset of $L^{2}\left(\Omega ; \mathbb{R}^{2}\right) \times L^{2}(\Omega)$, the Hahn-Banach theorem (see [4]) yields that there exist an element $(\boldsymbol{\theta}, \eta) \in L^{2}\left(\Omega ; \mathbb{R}^{2}\right) \times L^{2}(\Omega)$ and a real number $\gamma$ such that

$$
\int_{\Omega} \boldsymbol{p}_{0} \cdot \boldsymbol{\theta} \mathrm{d} \Omega+\int_{\Omega} \boldsymbol{\nabla} \cdot \boldsymbol{p}_{0} \eta \mathrm{d} \Omega<\gamma<\int_{\Omega} \boldsymbol{\phi} \cdot \boldsymbol{\theta} \mathrm{d} \Omega+\int_{\Omega} \boldsymbol{\nabla} \cdot \boldsymbol{\phi} \eta \mathrm{d} \Omega, \quad \forall \boldsymbol{\phi} \in \mathcal{K} .
$$

Since $\mathcal{K}$ is a convex cone whose vertex is zero, property (56) implies

$$
\int_{\Omega} \boldsymbol{p}_{0} \cdot \boldsymbol{\theta} \mathrm{d} \Omega+\int_{\Omega} \boldsymbol{\nabla} \cdot \boldsymbol{p}_{0} \eta \mathrm{d} \Omega<0 \leq \int_{\Omega} \boldsymbol{\phi} \cdot \boldsymbol{\theta} \mathrm{d} \Omega+\int_{\Omega} \eta \boldsymbol{\nabla} \cdot \boldsymbol{\phi} \mathrm{d} \Omega, \quad \forall \boldsymbol{\phi} \in \mathcal{K} .
$$

If not, the existence of $\phi$ such that

$$
\int_{\Omega} \phi \cdot \theta \mathrm{d} \Omega+\int_{\Omega} \eta \nabla \cdot \phi \mathrm{d} \Omega<0
$$

would give

$$
t\left\{\int_{\Omega} \boldsymbol{\phi} \cdot \boldsymbol{\theta} \mathrm{d} \Omega+\int_{\Omega} \eta \nabla \cdot \boldsymbol{\phi} \mathrm{d} \Omega\right\}<0
$$

for all $t>0$ and would lead to a contradiction. The subspace $\mathcal{D}\left(\Omega ; \mathbb{R}^{2}\right)$ is contained in $\mathcal{K}$. It follows then from (57) that

$$
\int_{\Omega} \boldsymbol{\phi} \cdot \boldsymbol{\theta} \mathrm{d} \Omega+\int_{\Omega} \eta \boldsymbol{\nabla} \cdot \boldsymbol{\phi} \mathrm{d} \Omega=0, \quad \forall \boldsymbol{\phi} \in \mathcal{D}\left(\Omega ; \mathbb{R}^{2}\right) .
$$


It then follows that

$$
\boldsymbol{\theta}=\nabla \eta
$$

Repeating similar arguments and making use of the fact that the subspace consisting of that $\phi \in \mathcal{D}\left(\bar{\Omega} ; \mathbb{R}^{2}\right)$, which vanish in a neighborhood of $\bar{\Gamma}_{N} \cup \bar{\Gamma}_{C}$, is included in $\mathcal{K}$, we also get that $\eta=0$ on $\Gamma_{D}$. Green formula permits us to check that $\eta \geq 0$ on $\Gamma_{C}$. Since $\boldsymbol{p}_{0} \in \boldsymbol{K}$, condition (57) leads to a contradiction. This ends the proof of the lemma.

Let us now introduce some notations. For $T \in \mathcal{T}_{h}, T^{\prime}$ will denote any edge of the triangle $T$. The set-wise non-disjoint union of all edges of $\mathcal{T}_{h}$ is denoted by $\mathcal{S}_{h}$. Recall that when $\boldsymbol{q} \in H(\operatorname{div} ; \Omega)$ is sufficiently regular, its interpolate $\mathcal{E}_{h} \boldsymbol{q}$ by the Raviart-Thomas finite element of the lowest degree is given by the following identification

$$
\left\{\begin{array}{l}
\mathcal{E}_{h} \boldsymbol{q} \in \boldsymbol{X}_{h} \\
\int_{T^{\prime}} \mu \mathcal{E}_{h} \boldsymbol{q} \cdot \boldsymbol{n} \mathrm{d} T^{\prime}=\int_{T^{\prime}} \mu \boldsymbol{q} \cdot \boldsymbol{n} \mathrm{d} T^{\prime}, \quad \forall T^{\prime} \in \mathcal{S}_{h}, \forall \mu \in \mathbb{P}_{0},
\end{array}\right.
$$

(see [23]). Moreover, the following estimates hold for the related approximation error

$$
\begin{gathered}
\left\|\boldsymbol{q}-\mathcal{E}_{h} \boldsymbol{q}\right\|_{0, \Omega} \leq C h|\boldsymbol{q}|_{1, \Omega}, \\
\left\|\boldsymbol{\nabla} \cdot\left(\boldsymbol{q}-\mathcal{E}_{h} \boldsymbol{q}\right)\right\|_{0, \Omega} \leq C h|\boldsymbol{\nabla} \cdot \boldsymbol{q}|_{1, \Omega} .
\end{gathered}
$$

Similarly, the $L^{2}$-orthogonal projection $P_{h}: L^{2} \longrightarrow M_{h}$ satisfies

$$
\left\|u-P_{h} u\right\|_{0, \Omega} \leq C h|u|_{1, \Omega}
$$

From Lemma 4.2, we can straightforwardly draw the following result.

Lemma 4.3. The discrete convex cone $\boldsymbol{K}_{h}$ approaches $\boldsymbol{K}$.

As a consequence, we get the convergence of the mixed finite method. We complete this result by proving a quasi-optimal error estimate under some regularity properties assumed on $u, \boldsymbol{p}$ and on the data $f$. These assumptions are reasonable in the sense that they are effectively satisfied when $\Omega$ is a convex domain, the interior PDE is the Laplacian and $\Gamma_{C}$ lies on a straight part of $\partial \Omega$ [21].

Theorem 4.4. Solution $\left(\boldsymbol{p}_{h}, u_{h}\right)$ to problem (51) converges strongly in $H(\operatorname{div} ; \Omega) \times L^{2}(\Omega)$ to the unique solution $(\boldsymbol{p}, u)$ of problem (5). Furthermore, if $\left.u\right|_{\Gamma_{C}} \in H^{1}\left(\Gamma_{C}\right), \boldsymbol{p} \cdot \boldsymbol{n} \in H^{\nu}\left(\Gamma_{C}\right)$ for $0<\nu<1, \boldsymbol{p} \in H^{1}\left(\Omega ; \mathbb{R}^{2}\right)$ and $f \in H^{1}(\Omega)$, there exists a constant $C>0$ independent of $h$ such that

$$
\left\|\boldsymbol{p}-\boldsymbol{p}_{h}\right\|_{H(\operatorname{div} ; \Omega)}+\left\|u-u_{h}\right\|_{0, \Omega} \leq C h^{(1+\nu) / 2}\left(\|u\|_{1, \Omega}+\|u\|_{1, \Gamma_{C}}+\|\boldsymbol{p}\|_{1, \Omega}+\|\boldsymbol{p} \cdot \boldsymbol{n}\|_{\nu, \Gamma_{C}}+\|f\|_{1, \Omega}\right) .
$$

Proof. The convergence of $\left(\boldsymbol{p}_{h}, u_{h}\right)$ is a simple induction based on Theorem 2.14, Lemma 4.3 and the standard approximation properties of $M_{h}$.

The rest of the proof requires several steps.

First, we establish the following estimate

$$
\inf _{\boldsymbol{q}_{h} \in \boldsymbol{K}_{h}}\left\{\left\|\boldsymbol{p}-\boldsymbol{q}_{h}\right\|_{H(\mathrm{div} ; \Omega)}^{2}+\left\langle\left(\boldsymbol{q}_{h}-\boldsymbol{p}\right) \cdot \boldsymbol{n}, u\right\rangle_{1 / 2, \partial \Omega}\right\} \leq C h^{1+\nu}\left(\|u\|_{1, \Omega}+\|\boldsymbol{p}\|_{1, \Omega}+\|\boldsymbol{\nabla} \cdot \boldsymbol{p}\|_{1, \Omega}\right)^{2} .
$$

The regularity of $u$ and that assumed of $\boldsymbol{p}$, as well as the boundary conditions, allow us to write

$$
\left\langle\left(\boldsymbol{q}_{h}-\boldsymbol{p}\right) \cdot \boldsymbol{n}, u\right\rangle_{1 / 2, \partial \Omega}=\int_{\Gamma_{C}} u\left(\boldsymbol{q}_{h}-\boldsymbol{p}\right) \cdot \boldsymbol{n} \mathrm{d} \Gamma_{C} .
$$


Indeed, we have

$$
\inf _{\boldsymbol{q}_{h} \in \boldsymbol{K}_{h}}\left\{\left\|\boldsymbol{p}-\boldsymbol{q}_{h}\right\|_{H(\mathrm{div} ; \Omega)}^{2}+\int_{\Gamma_{C}} u\left(\boldsymbol{q}_{h}-\boldsymbol{p}\right) \cdot \boldsymbol{n} \mathrm{d} \Gamma_{C}\right\} \leq\left\|\boldsymbol{p}-\mathcal{E}_{h} \boldsymbol{p}\right\|_{H(\mathrm{div} ; \Omega)}^{2}+\int_{\Gamma_{C}} u\left(\mathcal{E}_{h} \boldsymbol{p}-\boldsymbol{p}\right) \cdot \boldsymbol{n} \mathrm{d} \Gamma_{C}
$$

Since $\boldsymbol{\nabla} \cdot \boldsymbol{p}+f=0$, the bound on the first term in the right-hand side is given by (59) and (60)

$$
\left\|\boldsymbol{p}-\mathcal{E}_{h} \boldsymbol{p}\right\|_{H(\operatorname{div} ; \Omega)} \leq C h\left(|\boldsymbol{p}|_{1, \Omega}+|\boldsymbol{\nabla} \cdot \boldsymbol{p}|_{1, \Omega}\right)
$$

To get an estimate of $\int_{\Gamma_{C}} u\left(\mathcal{E}_{h} \boldsymbol{p}-\boldsymbol{p}\right) \cdot \boldsymbol{n} \mathrm{d} \Gamma_{C}$, we introduce the following discrete space

$$
Y_{h}=\left\{\mu_{h} \in L^{2}(\partial \Omega) ;\left.\quad \mu_{h}\right|_{T^{\prime}} \in \mathbb{P}_{0}, \quad \forall T^{\prime} \in \mathcal{S}_{h} \text { such that } T^{\prime} \subset \partial \Omega, \quad \mu_{h}=0 \text { on } \Gamma_{D}\right\}
$$

From the very definition of the degrees of freedom for the Raviart-Thomas finite elements of the lowest degree, we get

$$
\int_{T^{\prime}} \mu_{h} \boldsymbol{p} \cdot \boldsymbol{n} \mathrm{d} T^{\prime}=\int_{T^{\prime}} \mu_{h} \mathcal{E}_{h} \boldsymbol{p} \cdot \boldsymbol{n} \mathrm{d} T^{\prime}, \quad \forall T^{\prime} \subset \partial \Omega \forall \mu_{h} \in Y_{h}
$$

Hence, we can write

$$
\begin{aligned}
\int_{\Gamma_{C}} u\left(\mathcal{E}_{h} \boldsymbol{p}-\boldsymbol{p}\right) \cdot \boldsymbol{n} \mathrm{d} \Gamma_{C} & =\int_{\Gamma_{C}}\left(u-\mu_{h}\right)\left(\mathcal{E}_{h} \boldsymbol{p}-\boldsymbol{p}\right) \cdot \boldsymbol{n} \mathrm{d} \Gamma_{C} \\
& \leq\left\|u-\mu_{h}\right\|_{0, \Gamma_{C}}\left\|\left(\boldsymbol{p}-\mathcal{E}_{h} \boldsymbol{p}\right) \cdot \boldsymbol{n}\right\|_{0, \Gamma_{C}}
\end{aligned}
$$

Now, choosing $\mu_{h}$ as the projection $\pi_{h}$ of $\left.u\right|_{\partial \Omega}$ on $Y_{h}$, we get (cf. [9])

$$
\left\|u-\pi_{h} u\right\|_{0, \Gamma_{C}} \leq C h\|u\|_{1, \Gamma_{C}}
$$

In the same way, since $\left.\mathcal{E}_{h}(\boldsymbol{p} \cdot \boldsymbol{n})\right|_{\Gamma_{C}}$ is the projection of $\boldsymbol{p} \cdot \boldsymbol{n}$ on $Y_{h}$, the following bound holds

$$
\left\|\left(\boldsymbol{p}-\mathcal{E}_{h} \boldsymbol{p}\right) \cdot \boldsymbol{n}\right\|_{0, \Gamma_{C}} \leq C h^{\nu}\|\boldsymbol{p} \cdot \boldsymbol{n}\|_{\nu, \Gamma_{C}} .
$$

In view of (61), (64), (65) and (66), Proposition 4.1 yields

$$
\left\|\boldsymbol{p}-\boldsymbol{p}_{h}\right\|_{H(\operatorname{div} ; \Omega)}^{2} \leq C\left(h^{1+\nu}\|u\|_{1, \Gamma_{C}}\|\boldsymbol{p}\|_{1, \Gamma_{C}}+h^{2}\left(\|\boldsymbol{p}\|_{1, \Omega}^{2}+\|\boldsymbol{\nabla} \cdot \boldsymbol{p}\|_{1, \Omega}^{2}\right)\right),
$$

and

$$
\left\|u-u_{h}\right\|_{0, \Omega} \leq C\left(\left\|\boldsymbol{p}-\boldsymbol{p}_{h}\right\|_{H(\operatorname{div} ; \Omega)}+h|u|_{1, \Omega}\right) .
$$

The rest of the proof is straightforward.

Estimate (62) indicates a loss of order $h^{(1-\nu) / 2}$ relatively to the optimal error estimate for the lowest order Raviart-Thomas finite element method. Now we wish to examine whether this loss is simply a consequence of the technique of proof or really relies upon a feature related to the nonlinear character of the problem.

Assuming that $u$ is at least in $\mathcal{C}^{0}\left(\overline{\Gamma_{C}}\right)$, we define

$$
\mathcal{S}_{h, C}:=\left\{T^{\prime} \in \mathcal{S}_{h} ; T^{\prime} \subset \Gamma_{C}, u(x)=0 \text { and } u(y)>0 \text { at two interior points } x \text { and } y \text { of } T^{\prime}\right\},
$$

that is, the set of edges $T^{\prime}$ where $u$ changes from the Dirichlet to the Neumann boundary condition. The number of such edges is denoted by $\mathcal{N}_{h, C}$. This makes it possible to express the contribution of the unilateral boundary condition in a more accurate way assuming once more that $u$ owns the regularity properties of the solutions to Signorini's problem in polygonal domains. 
Lemma 4.5. Assume that $u \in H^{1 / 2+\mu}\left(\Gamma_{C}\right)$ for $0<\mu<1$ and $\boldsymbol{p} \cdot \boldsymbol{n} \in H^{\nu}\left(\Gamma_{C}\right)$ for $0<\nu<1$, then there exists a constant $C$ independent of $h$ such that

$$
\left|\int_{\Gamma_{C}} u\left(\mathcal{E}_{h} \boldsymbol{p}-\boldsymbol{p}\right) \cdot \boldsymbol{n} \mathrm{d} \Gamma_{C}\right| \leq C h^{\mu+\nu}\left(\mathcal{N}_{h, C} h\right)^{1 / 2}\|u\|_{1 / 2+\mu, \Gamma_{C}}\|\boldsymbol{p} \cdot \boldsymbol{n}\|_{\nu, \Gamma_{C}} .
$$

Proof. The first ingredient is to remark that the integral in the left-hand side of (68) is reduced to the edges $T^{\prime} \in \mathcal{S}_{h, C}$ since either $u$ or $\boldsymbol{p} \cdot \boldsymbol{n}$ vanishes everywhere else. Thus, Cauchy-Schwarz inequality and estimate (66) reduce the proof to a bound for

$$
\left(\sum_{T^{\prime} \in \mathcal{S}_{h, C}} \int_{T^{\prime}}|u(x)|^{2} \mathrm{~d} T^{\prime}\right)^{1 / 2}
$$

Taking into account that $u$ vanishes at some point $y$ of $T^{\prime} \in \mathcal{S}_{h, C}$ and making use of the embedding theorems of Sobolev into Holder spaces ( $c f .[1])$, we get

$$
|u(x)|=|u(x)-u(y)| \leq C|x-y|^{\mu}\|u\|_{1 / 2+\mu, \Gamma_{C}} \leq C h^{\mu}\|u\|_{1 / 2+\mu, \Gamma_{C}}, \forall x \in T^{\prime}, \forall T^{\prime} \in \mathcal{S}_{h, C} .
$$

The rest of the proof is straightforward.

Remark 4.6. Without any further information on the solution $u$, we can only say that $\mathcal{N}_{h, C} h$ is bounded uniformly in $h$ so getting quasi-optimal estimate (62) from somewhat different assumptions and with more intricate proofs. However the following theorem makes it possible to obtain an optimal error estimate under an assumption usually assumed on the solutions of unilateral boundary-value problems.

Theorem 4.7. Under the general conditions of Theorem 4.4 and Lemma 4.5, moreover if there is only a finite number of points in $\Gamma_{C}$ where $u$ changes from a Dirichlet to a Neumann condition and $\mu$ and $\nu$ are such that $\mu+\nu \geq 3 / 2$, then the error satisfies the following optimal bound

$$
\left\|\boldsymbol{p}-\boldsymbol{p}_{h}\right\|_{H(\operatorname{div} ; \Omega)}+\left\|u-u_{h}\right\|_{0, \Omega} \leq C h\left(\|u\|_{1, \Omega}+\|u\|_{\mu, \Gamma_{C}}+\|\boldsymbol{p}\|_{1, \Omega}+\|\boldsymbol{p} \cdot \boldsymbol{n}\|_{\nu, \Gamma_{C}}+\|f\|_{1, \Omega}\right),
$$

with a constant $C$ independent of $h$.

Proof. The assumption on the changes of the boundary conditions satisfied by $u$ implies that $\mathcal{N}_{h, C}$ is bounded independently of $h$. The nonlinear contribution to the error is hence bounded by

$$
C h^{\mu+\nu+1 / 2}\|u\|_{1 / 2+\mu, \Gamma_{C}}\|\boldsymbol{p} \cdot \boldsymbol{n}\|_{\nu, \Gamma_{C}}
$$

The rest of the proof is elementary.

Remark 4.8. In the same way, Theorems 3.9 and 4.7 yield that the following discrete problem

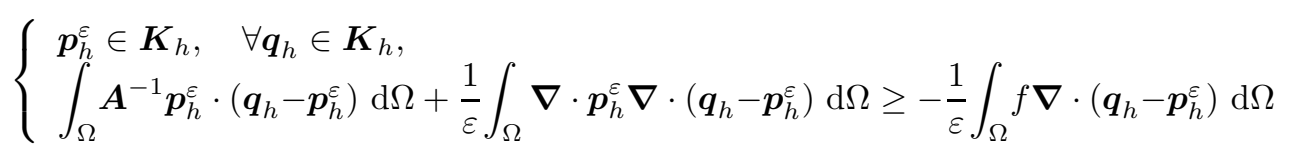

where $\boldsymbol{K}_{h}$ is the above approximation of the convex cone $\boldsymbol{K}$ by the mixed Raviart-Thomas finite element method of the lowest order has a solution converging in a uniform way relatively to $\varepsilon$ with respectively a quasi-optimal and an optimal error bound.

Acknowledgements. The authors would like to acknowledge the thorough reading of the referees and their helpful remarks. 


\section{REFERENCES}

[1] D.A. Adams, Sobolev spaces. Academic Press, New York (1975).

[2] L. Baillet and T. Sassi, Méthode d'éléments finis avec hybridisation frontière pour les problèmes de contact avec frottement. C.R. Acad. Sciences Paris Série I 334 (2002) 917-922.

[3] F. Ben Belgacem, Y. Renard and L. Slimane, A mixed formulation for the Signorini problem in incompressible elasticity, theory and finite element approximation. Appl. Numer. Math. (to appear).

[4] H. Brezis, Analyse fonctionnelle: Théorie et applications. Masson, Paris (1983).

[5] F. Brezzi and M. Fortin, Mixed and hybrid finite element methods. Springer-Verlag, Berlin (1991).

[6] F. Brezzi, W. Hager and P.A. Raviart, Error estimates for the finite element solution of variational inequalities, Part II. Numer. Math 31 (1978) 1-16.

[7] D. Capatina-Papaghiuc, Contribution à la prévention de phénomènes de verrouillage numérique. Ph.D. thesis, Université de Pau, France (1997).

[8] D. Capatina-Papaghiuc and N. Raynaud, Numerical approximation of stiff transmission problems by mixed finite element methods. RAIRO Modél. Math. Anal. Numér. 32 (1998) 611-629.

[9] P.G. Ciarlet, The finite element methods for elliptic problems. North-Holland, Amsterdam (1978).

[10] P. Coorevits, P. Hild, K. Lhalouani and T. Sassi, Mixed finite elemen methods for unilateral problems: convergence analysis and numerical studies. Math. Comp. 71 (2001) 1-25.

[11] G. Duvaut and J.-L. Lions, Les inéquations en mécanique et en physique. Dunod, Paris (1972).

[12] I. Ekeland and R. Temam, Analyse convexe et problèmes variationnels. Dunod, Paris (1974).

[13] R.C. Falk, Error estimates for the approximation of a class of variational inequalities. Math. Comp. 28 (1974) 863-971.

[14] J. Haslinger, Mixed formulation of elliptic variational inequalities and its approximation. Appl. Math. 6 (1981) $462-475$.

[15] J. Haslinger, I. Hlaváček and J. Nečas, Numerical methods for unilateral problems in solid mechanics. Handb. Numer. Anal., Vol. IV: Finite Element Methods, Part 2 - Numerical Methods for solids, Part 2, P.G. Ciarlet and J.-L. Lions Eds., NorthHolland, Amsterdam (1996).

[16] J. Jarušek, Contact problems with bounded friction, coercive case. Czech. Math. J. 33 (1983) $237-261$.

[17] N. Kikuchi and J.T. Oden, Contact problems in elasticity: A Study of variational Inequalities and Finite Element Methods. SIAM, Philadelphia (1988).

[18] K. Lhalouani and T. Sassi, Nonconforming mixed variational formulation and domain decomposition for unilateral problems. East-West J. Num. Math. 7 (1999) 23-30.

[19] J.-L. Lions, Quelques méthodes de résolution de problème aux limites non linéaires. Dunod, Paris (1969).

$[20]$ U. Mosco, Convergence of convex sets and of solutions of variational inequalities. Adv. Math. 3 (1969) 510-585.

[21] M. Moussaoui and K. Khodja, Régularité des solutions d'un problème mêlé Dirichlet-Signorini dans un domaine polygonal plan. Comm. Partial Differential Equations 17 (1992) 805-826.

[22] N. Raynaud, Approximation par méthode d'éléments finis de problèmes de transmission raides. Ph.D. thesis, Université de Pau, France (1994).

[23] J.E. Robert and J.-M. Thomas, Mixed and Hybrid Methods. Handb. Numer. Anal., Vol. II: Finite Element Methods, Part 1, North-Holland, Amesterdam (1991).

[24] L. Slimane, Méthodes mixtes et traitement du verrouillage numérique pour la résolution des inéquations variationnelles. $\mathrm{Ph} . \mathrm{D}$. thesis, INSA de Toulouse, France (2001).

[25] L. Slimane, A. Bendali and P. Laborde, Mixed formulations for a class of variational inequalities. C.R. Math. Acad. Sci. Paris 334 (2002) 87-92.

[26] L. Wang and G. Wang, Dual mixed finite element method for contact problem in elasticity. Math. Num. Sin. 21 (1999).

To access this journal online:

www.edpsciences.org 